\title{
Spectroscopic monitoring of the luminous blue variable Westerlund1-243 from 2002 to 2009*
}

\author{
B. W. Ritchie ${ }^{1,2}$, J. S. Clark ${ }^{1}$, I. Negueruela ${ }^{3}$, and F. Najarro ${ }^{4}$ \\ 1 Department of Physics and Astronomy, The Open University, Walton Hall, Milton Keynes MK7 6AA, UK \\ e-mail: b.ritchie@open.ac.uk \\ 2 IBM United Kingdom Laboratories, Hursley Park, Winchester, SO21 2JN, UK \\ 3 Departamento de Física, Ingeniería de Sistemas y Teoría de la Señal, Universidad de Alicante, Apdo. 99, 03080 Alicante, Spain \\ ${ }^{4}$ Laboratorio de Física Estelar y Exoplanetas, Centro de Astrobiología, CSIC-INTA, Ctra de Torrejón a Ajalvir km 4, \\ 28850 Torrejón de Ardoz, Spain
}

Received 25 July 2009 / Accepted 1 October 2009

\section{ABSTRACT}

\begin{abstract}
Context. The massive post-main sequence star W243 in the galactic starburst cluster Westerlund 1 has undergone a spectral transformation from a B2Ia supergiant devoid of emission features in 1981 to an A-type supergiant with a rich emission-line spectrum by 2002/03.

Aims. We examine the continued evolution of W243 from 2002 to 2009 to understand its evolutionary state, current physical properties and the origin of its peculiar emission line spectrum.

Methods. We used VLT/UVES and VLT/FLAMES to obtain high resolution, high signal-to-noise spectra on six epochs in 2003/04 (UVES) and ten epochs in 2008/09 (FLAMES). These spectra are used alongside other lower-resolution VLT/FLAMES and NTT/EMMI spectra to follow the evolution of W243 from 2002 to 2009. Non-LTE models are used to determine the physical properties of W243.

Results. W243 displays a complex, time-varying spectrum with emission lines of hydrogen, helium and Lyman- $\alpha$ pumped metals, forbidden lines of nitrogen and iron, and a large number of absorption lines from neutral and singly-ionized metals. Many lines are complex emission/absorption blends, with significant spectral evolution occurring on timescales of just a few days. The LBV has a

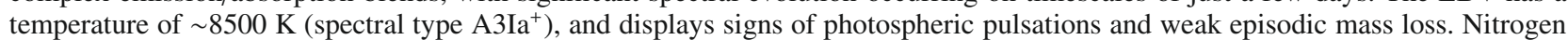
is highly overabundant, with carbon and oxygen depleted, indicative of surface CNO-processed material and considerable previous mass-loss, although current time-averaged mass-loss rates are low. The emission-line spectrum forms at large radii, when material lost by the LBV in a previous mass-loss event is ionized by an unseen hot companion. Monitoring of the near-infrared spectrum suggests that the star has not changed significantly since it finished evolving to the cool state, close to the Humphreys-Davidson limit, in early 2003.
\end{abstract}

Key words. stars: individual: W243 - stars: evolution - supergiants - stars: variables: general - stars: winds, outflows

\section{Introduction}

Luminous blue variables (LBVs; Humphreys \& Davidson 1994) represent a short-lived transitional stage in the life of the most massive stars as they leave the main sequence and evolve towards the Wolf-Rayet phase. LBVs are characterized by very high rates of mass-loss, dense, slow winds and variability in luminosity and temperature that range from short-term microvariations of $\sim 0.1$ to $0.2 \mathrm{mag}$ to the rare, catastrophic $\eta$ Carinae type eruptions for which the LBV class is most well known. In its quiescent state a LBV appears as a very luminous $\left(\sim 10^{6} L_{\odot}\right)$ B-type supergiant that lies on the $\mathrm{S}$ Doradus instability strip (Smith et al. 2004), but on timescales of a few decades the LBV will move redwards on the Hertzsprung-Russell diagram (HRD), taking on the appearance of an A- or F-type supergiant and brightening by $m_{v} \sim 1$ to 2 due to the change in bolometric correction. More rarely, LBVs may undergo giant eruptions

* This work is based on observations collected at the European Southern Observatory, Chile, under programme IDs ESO 69.D0039(A), 071.D-0151(A), 271.D-5045(A)， 073.D-0025(A ...C), 075.D-0388(A ... C), 081.D-0324(A ...F) and 383.D-0633(A). in which bolometric luminosity is not conserved and substantial mass-loss occurs, with many LBVs surrounded by expanding nebule (e.g. Weis 2003) that consist of material ejected from the star during earlier mass-loss events. Finally, there is growing evidence that LBVs may be the progenitors of some type IIn supernovae (e.g. SN2006gy, Smith et al. 2007; SN 2005gl, Gal-Yam \& Leonard 2009), while the bipolar nebula surrounding the candidate-LBV HD 168625 has a morphology similar to Sk $-69^{\circ} 202$, the progenitor of SN1987A (Smith 2007). However, the rarity of LBVs means that the nature of these stars is still poorly understood, and the mechanisms behind the periodic increases in mass loss as the star leaves the quiescent state and the causes of the giant $\eta$-Carinae type eruptions are unknown.

A recent addition to the catalogue of galactic LBVs is W243 ${ }^{1}$ (Clark \& Negueruela 2004, hereafter Paper I) in the starburst cluster Westerlund 1 (hereafter Wd 1; Westerlund 1961; Clark et al. 2005). Early observations included star $G(=\mathrm{W} 243)$ amongst a group of OB supergiants, with Borgman et al. (1970)

\footnotetext{
${ }^{1} \mathrm{RA}=164707.5 \delta=-455228.5, \mathrm{~J} 2000$.
} 
reporting $m_{v} \sim 15.1 \pm 0.2^{2}$ and spectral type B0.5Ia, while Lockwood (1974) list $m_{v} \sim 15.6$; see also Koorneef (1977). Westerlund (1987) report $m_{v} \sim 14.38$ from photometry obtained in 1960-66, but used spectroscopy obtained in 1981 to classify W243 as a B2Ia supergiant with a spectrum devoid of emission lines. However, Paper I found that by 2002/03 W243 was displaying the spectrum of an A2Ia supergiant, implying an apparent decrease in temperature of $\sim 10^{4} \mathrm{~K}$ during the intervening period, accompanied by the development of a rich emission line spectrum showing lines of $\mathrm{H}$, He I, and $\mathrm{Ca}$ II. Excellent agreement was found between the spectral types of six other Band A-type supergiants observed by both Westerlund (1987) and Clark et al. (2005), and Paper I concluded that W243 is an LBV that was quiescent when observed by Westerlund (1987) but has since undergone a significant outburst, moving it redwards on the H-R diagram and (assuming constant bolometric luminosity) close to the Humphreys-Davidson limit.

More recently, W243 has been listed as an aperiodic variable with $m_{v} \sim 15.73$ by Bonanos (2007), while Groh et al. (2006, 2007) report infrared spectra with Pa $\gamma$ emission that resembles the cool phase of the LBV HR Carinae (Machado et al. 2002) and a $K$-band spectral morphology that is very similar to other LBVs such as AG Carinae (Groh et al. 2009), LBV 1806-20 (Eikenberry et al. 2004) and the Pistol star (Figer et al. 1998). However, the strong He I emission seen at $6678 \AA$, $7065 \AA$ (Paper I) and $10830 \AA$ (Groh et al. 2007) is anomalous, and Groh et al. (2006) note that the $K$-band spectrum also implies a higher temperature than that of a typical yellow hypergiant (YHG) and suggest that W243 may be evolving back towards a hotter state. Clark et al. (2008) report W243 as a weak $\left(L_{\mathrm{x}}<10^{32} \mathrm{erg} \mathrm{s}^{-1}\right)$ $X$-ray source ${ }^{3}$, while Dougherty et al. (2009) find a current massloss rate for $\mathrm{W} 243$ of $4-6 \times 10^{-6} M_{\odot} \mathrm{yr}^{-1}$ from radio observations. No evidence is seen of the nebulosity typically surrounding LBVs (e.g. Weis 2003), although it is likely that such a nebula would be rapidly disrupted in the environment of $\mathrm{Wd} 1$. Finally, Ritchie et al. (2009) report evidence for photospheric pulsations in W243; this is examined further in Sect. 3.2.2.

This paper presents further analysis of the Paper I dataset, along with analysis of five subsequent high-resolution echelle spectra taken during 2004 and intermediate- and high-resolution spectra from 2005, 2008 and 2009 to examine the physical state and evolution of W243 over a seven-year timescale. Details of the observations and data reduction are presented in Sect. 2, results are presented in Sect. 3 and are discussed in Sect. 4.

\section{Observations and data reduction}

Observations of W243 were made on six nights in 2003 and 2004 using the Ultraviolet and Visual Echelle Spectrograph (UVES; Dekker et al. 2000) located at the Nasmyth B focus of the $8.2 \mathrm{~m}$ VLT UT2 Kueyen at Cerro Paranal, Chile. UVES was used in RED mode with cross-dispersers CD\#3 (4760-6840 ̊) and CD\#4 (6600-10600 ̊), and in DICHR\#1 mode with crossdisperser CD\#3 (4600-6680 А) for the red arm. All modes give $R \sim 40000$. Due to the strength of the $\mathrm{H} \alpha$ emission from

\footnotetext{
2 We note that photometry from different studies of Wd1 tends to show systematic differences on the order of a few tenths of a magnitude (Clark et al. 2009, in prep.) and the values for W243 listed here may not be directly comparable.

3 This is consistent with X-ray observations of other LBVs (e.g. Muno et al. 2006), although some LBVs (e.g. $\eta$ Carinae, HD 5980) have significantly higher X-ray luminosities (Muno et al. 2006; Clark et al. 2008)
}

W243, two $R$-band spectra were taken; a $500 \mathrm{~s}$ integration to capture the $\mathrm{H} \alpha$ line, and a longer $1800 \mathrm{~s}$ integration (2750 s on $21 / 09 / 2003, \mathrm{MJD}=52903.0)$ in which the $\mathrm{H} \alpha$ line is saturated but the signal-to-noise $(S / N)$ ratio of the remaining features is improved. The $S / N$ ratio is in excess of 100 at $\lambda>6200 \AA$ for the long RED and DICHR\#1 mode integrations, with a minimum on the UVES REDU CCD of $\sim 77$ at $~ 5850 \AA$, dropping below 50 at $\sim 5500 \AA$ on the REDL CCD. The $S / N$ ratio averages $\sim 160$ for the integrations centred at $860 \mathrm{~nm}$. Due to the high degree of reddening towards $\mathrm{Wd} 1\left(A_{v} \sim 12\right.$; Clark et al. 2005) the $S / N$ ratio of data from the blue arm in DICHR\#1 mode (centred at $390 \mathrm{~nm}$ ) is very poor. The data were bias subtracted, flat fielded and wavelength calibrated using the UVES data reduction pipeline ${ }^{4}$, version 3.9.0, with version 4.1.0 of the Common Pipeline Library (CPL).

In addition to the UVES spectra, observations were made on three nights in 2005, eight nights in 2008 and two nights in 2009 using the Fibre Large Array Multi Element Spectrograph (FLAMES; Pasquini et al. 2002), located at the Nasmyth A focus of VLT UT2. FLAMES was used with the GIRAFFE spectrograph in MEDUSA mode, with the 2005 observations using LR6 and LR8 to cover 6438-7184 $\AA$ and 8206-9400 with $R \sim 8600$ and $\sim 6500$ respectively, and the 2008 observations using HR21 to cover the 8484-9001 $\AA$ range with $R \sim 16200.600 \mathrm{~s}$ integrations were used, giving a $S / N$ ratio in excess of 100 . The FLAMES data were reduced in a similar manner to the UVES data, using version 2.5.3 of the FLAMES-GIRAFFE pipeline with individual spectra extracted using the IRAF ${ }^{5}$ task onedspec. Finally, we make use of ESO Multi-Mode Instrument (EMMI; Dekker et al. 1986) spectra covering the 8225-8900 $\AA$ region from 06/2002 and 06/2003 (see Paper I), and a NTT/EMMI spectrum from 02/2006 covering the 6310-7835 $\AA$ region. These were obtained using the NTT at La Silla, Chile, and data reduction is described in Paper I.

A summary of all the observations is given in Table 1. Analysis made use of packages within the Starlink software suite, including FIGARO (Shortridge et al. 1997) and DIPSO (Howarth et al. 2003). Equivalent widths were measured using the DIPSO $E W$ command and integrating the flux relative to a linear continuum based on estimated points on either side of the line. Radial velocities were calculated by correcting for heliocentric velocity and then fitting Gaussian profiles to the absorption features using IRAF task ngaussfit. In general this produced a satisfactory fit, but a number of strong lines display asymmetric wings and the measurement of the line centre was refined by carrying out a second fit to the core of the line profile, excluding the asymmetric component of the absorption line.

\section{Description of the spectra}

The VLT/UVES and VLT/FLAMES spectra of W243 reveal a complex, time-varying spectrum containing absorption and emission features, as well as absorption/emission blends with P Cygni, inverse P Cygni and double-peaked profiles: the region around the $\mathrm{H} \alpha$ line is plotted in Fig. 1, with principal features marked. Over the entire spectrum, strong emission is seen from the $\mathrm{H} \alpha$ and $\mathrm{H} \beta$ Balmer series lines, the $\mathrm{Pa} \delta \lambda 10049$

\footnotetext{
4 http://wwW.eso.org/sci/data-processing/software/ pipelines/

5 IRAF is distributed by the National Optical Astronomy Observatories, which are operated by the Association of Universities for Research in Astronomy, Inc., under cooperative agreement with the National Science Foundation.
} 
Table 1. Dates of observations, instruments and configurations used.

\begin{tabular}{|c|c|c|c|}
\hline Date & MJD & Instrument & Configuration $^{a}$ \\
\hline $07 / 06 / 2002$ & 52432.2 & EMMI & REMD, $2 \times 2$, GRAT7 \\
\hline $06 / 06 / 2003$ & 52796.0 & EMMI & REMD, $2 \times 2$, GRAT7 \\
\hline $07 / 06 / 2003$ & 52797.1 & EMMI & REMD, $1 \times 1$, GRAT6 \\
\hline $21 / 09 / 2003$ & 52903.0 & UVES & $\begin{array}{l}\text { RED, 580, CD3 } \\
\text { RED, 860, CD4 }\end{array}$ \\
\hline 03/04/2004 & 53098.4 & UVES & $\begin{array}{l}\text { RED, 580, CD3 } \\
\text { RED, 860, CD4 } \\
\text { DICHR1, 564, CD3 }\end{array}$ \\
\hline 09/06/2004 & 53165.2 & UVES & $\begin{array}{l}\text { RED, 580, CD3 } \\
\text { RED, 860, CD } 4 \\
\text { DICHR } 1,564, \text { CD3 }\end{array}$ \\
\hline 07/07/2004 & 53193.1 & UVES & DICHR1, 564, CD3 \\
\hline $10 / 07 / 2004$ & 53196.2 & UVES & $\begin{array}{l}\text { RED, 580, CD3 } \\
\text { RED, 860, CD4 } \\
\text { DICHR1, 564, CD3 }\end{array}$ \\
\hline 28/09/2004 & 53276.0 & UVES & $\begin{array}{l}\text { RED, 580, CD3 } \\
\text { RED, 860, CD4 } \\
\text { DICHR1, 564, CD3 }\end{array}$ \\
\hline $25 / 03 / 2005$ & 53454.3 & FLAMES & $\begin{array}{l}\text { MEDUSA, LR6 } \\
\text { MEDUSA, LR8 }\end{array}$ \\
\hline $29 / 05 / 2005$ & 53519.3 & FLAMES & $\begin{array}{l}\text { MEDUSA, LR6 } \\
\text { MEDUSA, LR8 }\end{array}$ \\
\hline $13 / 07 / 2005$ & 53564.1 & FLAMES & $\begin{array}{l}\text { MEDUSA, LR6 } \\
\text { MEDUSA, LR8 }\end{array}$ \\
\hline $17 / 02 / 2006$ & 53783.4 & EMMI & RILD, $1 \times 1$, GRISM6 \\
\hline $29 / 06 / 2008$ & 54646.2 & FLAMES & MEDUSA, HR21 \\
\hline $18 / 07 / 2008$ & 54665.0 & FLAMES & MEDUSA, HR21 \\
\hline $24 / 07 / 2008$ & 54671.1 & FLAMES & MEDUSA, HR 21 \\
\hline $14 / 08 / 2008$ & 54692.0 & FLAMES & MEDUSA, HR21 \\
\hline $04 / 09 / 2008$ & 54713.0 & FLAMES & MEDUSA, HR21 \\
\hline $15 / 09 / 2008$ & 54724.1 & FLAMES & MEDUSA, HR21 \\
\hline $19 / 09 / 2008$ & 54728.1 & FLAMES & MEDUSA, HR 21 \\
\hline $25 / 09 / 2008$ & 54734.1 & FLAMES & MEDUSA, HR 21 \\
\hline $14 / 05 / 2009$ & 54965.2 & FLAMES & MEDUSA, HR21 \\
\hline $18 / 05 / 2009$ & 54969.3 & FLAMES & MEDUSA, HR21 \\
\hline
\end{tabular}

a The VLT/UVES configurations list the instrument mode (RED or DICHR\#1), central wavelength (nm) and cross-disperser (CD\#3 or CD\#4). The NTT/EMMI configurations list the observing mode (REMD or RILD), binning and grating, while the VLT/FLAMES configurations list the GIRAFFE spectrograph mode and setup name.

and $\mathrm{Pa} \epsilon \lambda 9545$ Paschen series lines, He I $\lambda \lambda 5015,5876,6678$, 7065 and Ca II $\lambda \lambda 8498,8542,8662$. Weak emission is also seen from He I $\lambda \lambda 7281,8583$. Forbidden line emission is seen from [N II] $\lambda \lambda 5754,6584$ and [Fe II] $\lambda 7155$, while weaker [N II] $\lambda 6548$ and [Fe II] $\lambda \lambda 7388,7453$ lines are also seen. A possible [Fe II] $\lambda 4799$ line is seen at the extreme blue end of the UVES spectra. Very weak [S II] $\lambda \lambda 6716,6731$ emission is tentatively identified. Many Fe II lines are seen in both emission and absorption, with some lines displaying strong inverse $\mathrm{P}$ Cygni profiles in the $21 / 09 / 2003$ (MJD $=52903.0)$ spectrum, a feature also seen in the O I $\lambda 8446$ line and higher Paschen series lines. Strong absorption lines are seen from Si II $\lambda \lambda 6347$, 6371, the O I $\lambda 7774$ triplet and N I multiplet 1 and 8 lines in the near infra-red, while many weaker absorption lines from singlyionized iron group elements such as $\mathrm{Cr}$ II, Sc II and Ti II are also seen: a full list is given in Table A.1. Finally, the spectrum contains many interstellar absorption features, including diffuse interstellar bands, a very strong $\mathrm{Na}$ I $\lambda \lambda 5890,5896 \mathrm{dou}-$ blet and K I $\lambda 7699$.

Due to the high degree of reddening towards Wd 1 our analysis focuses predominantly on features redwards of $\sim 6000 \AA$ where the $S / N$ ratio is high and spectral features are clearly defined. The short-integration UVES RED arm, cross-disperser \#3 spectra were used to examine the $\mathrm{H} \alpha$ line, which is saturated in the longer UVES integrations. All other spectral features in the 4760-6650 $\AA$ region were examined using the long-integration UVES data. At longer wavelengths, UVES cross-disperser \#4 and FLAMES spectra were used. Unless noted in the text, rest wavelengths are taken from Moore (1945) or from the NIST Atomic Spectra Database ${ }^{6}$.

\subsection{Emission lines}

\subsubsection{Balmer- and Paschen-series emission}

Paper I reported strengthening $\mathrm{H} \alpha$ line emission throughout 2001-2003, a trend that continues into early 2004. The $\mathrm{H} \alpha$ line, plotted in the left panel of Fig. 2, displays a double-peaked profile in all UVES spectra, but the central absorption feature becomes less pronounced and the line strengthens predominantly in the redwards peak; by mid-2004 the bluewards peak is at virtually the same level as the 2003 spectrum. Broad electronscattering wings are visible, extending to $\pm 1000 \mathrm{~km} \mathrm{~s}^{-1}$. The $\mathrm{H} \beta$ line (not shown) also strengthens in early 2004 and displays a similar double-peaked profile with a central absorption feature blueshifted $50 \mathrm{~km} \mathrm{~s}^{-1}$ from its rest wavelength. Table 2 lists the equivalent width of the $\mathrm{H} \alpha$ line from each observation, along with the radial velocities of the two peaks, the central absorption feature and the separation between the two peaks in the profile. Lower resolution VLT/FLAMES spectra obtained in 2005 show a weaker but still clearly asymmetric $\mathrm{H} \alpha$ line profile but cannot resolve the central absorption feature seen in the UVES spectra, while by 2006 a NTT/EMMI spectrum shows that the $\mathrm{H} \alpha$ line has weakened further to 2002/03 levels; in this case the resolution is insufficient to see if the asymmetry is still present.

Paschen series lines are also seen in emission, with a strong $\operatorname{Pa} \delta \lambda 10049$ line (shown in the right panel of Fig. 2) and weakening emission from the Pa8 $\lambda 9546$ and Pa9 $\lambda 9229$ lines. Unlike the Balmer-series lines, the Paschen-series lines do not show an obvious double-peaked profile and the centre of the $\operatorname{Pa} \delta \lambda 10049$ line is almost constant between observations; values are again listed in Table 2 . The line strengthens and narrows slightly during 2004, but the changes in equivalent width are predominantly due to the variable absorption on both sides of the emission line. This is redshifted relative to the emission line in the 2003 spectrum, but is increasingly seen on the blue side of the line during 2004, with a pronounced $\mathrm{P}$ Cygni profile visible in the 10/07/2004 (MJD = 53 196.2) spectrum. The higher Paschenseries lines are seen predominantly in absorption, with the emission that dominates the $\operatorname{Pa} \delta$ line seen instead as weaker core emission that splits the blue wing of the absorption line; this can be seen in Fig. 3, which plots the Pa11 line over the course of the VLT/FLAMES observations. The absorption component shows notable variability (discussed further in Sect. 3.2) while the emission component is largely static, showing little change in strength or radial velocity over the course of our VLT/UVES and VLT/FLAMES spectra.

\subsubsection{He I emission}

He I $\lambda \lambda 5015,5876,6678,7065,7281$ and 8583 are seen in emission, with strong infra-red He I $\lambda 1.083 \mu \mathrm{m}$ and He I $\lambda 2.058 \mu \mathrm{m}$ emission lines also reported (Groh et al. 2006,

\footnotetext{
6 http://physics.nist.gov/PhysRefData/ASD/lines_form. html
} 

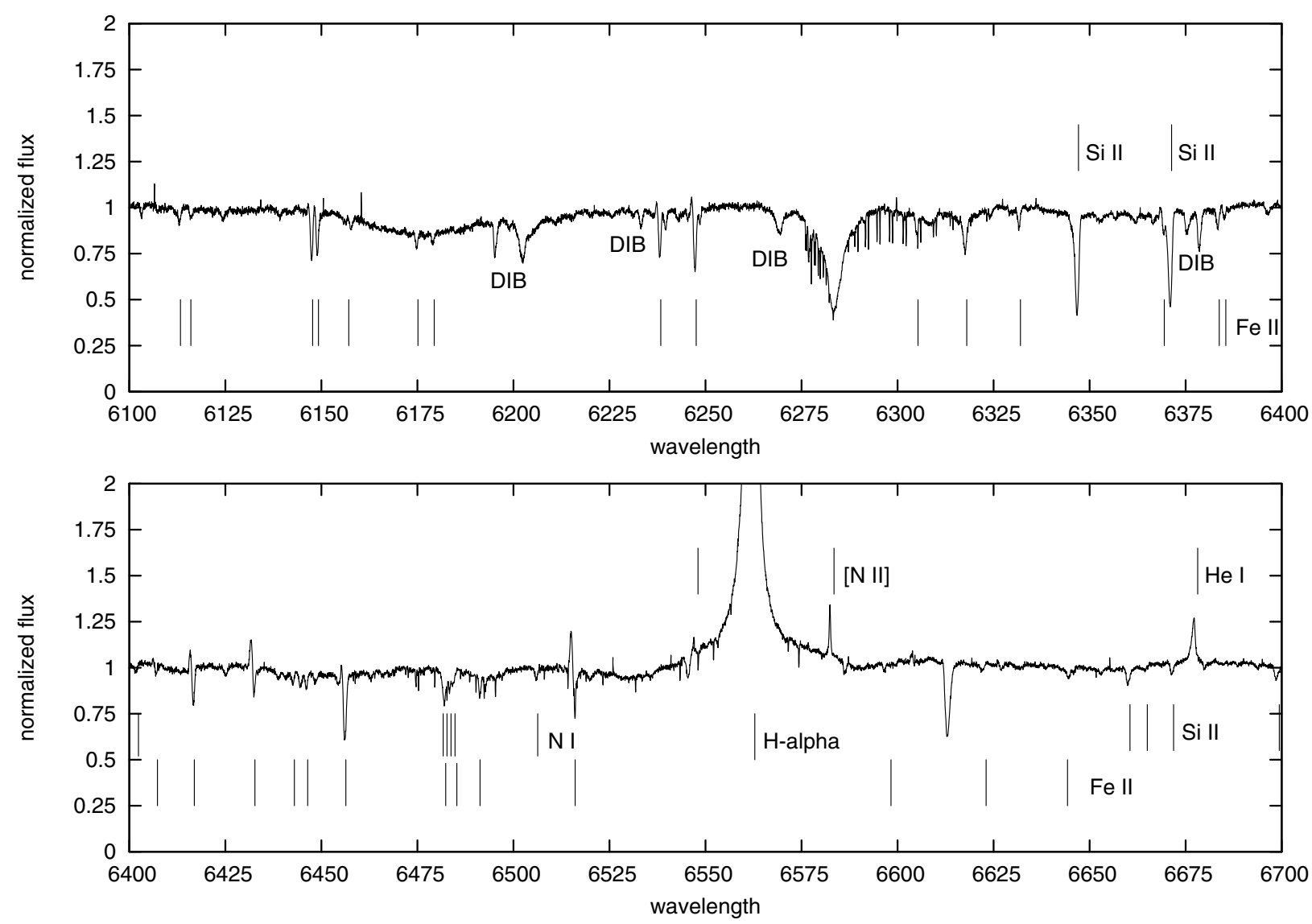

Fig. 1. VLT/UVES spectrum of W243 covering 6100-6700 $\AA$ from 21/09/2003 (MJD = 52 903.0) with principal features discussed in the text marked. Rest wavelengths are indicated. Identified diffuse interstellar bands are also marked.
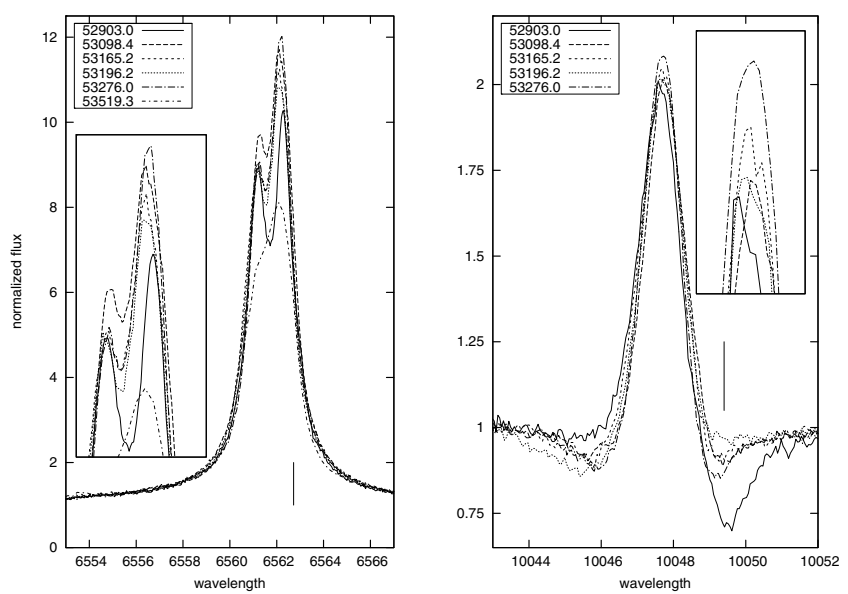

Fig. 2. Evolution of the H $\alpha$ (UVES + FLAMES, left panel) and $\mathrm{Pa} \delta \lambda 10049$ (UVES, right panel) emission lines. Insets show magnified views of the centre of the emission line, and rest wavelengths are marked in both panels.

2007). No He II features are detected. The triplet He I $\lambda 7065$ line is plotted in Fig. 4, with measurements of the equivalent width and radial velocity given in Table 2 . With the exception of the $21 / 09 / 2003$ (MJD = 52903.0) spectrum, the line shows a near-constant profile on the red side of the emission line with emission extending $\sim 100 \mathrm{~km} \mathrm{~s}^{-1}$ redwards of the line centre. The bluewards emission is more variable, decreasing substantially in the $03 / 04 / 2004(\mathrm{MJD}=53098.4)$ spectrum before recovering to earlier levels; this decrease, which clips the peak emission, is responsible for the decrease in measured radial velocity listed in Table 2. For other epochs, the measured radial velocity is very similar to that measured from the $\operatorname{Pa} \delta$ line, with any differences likely due to the blue-wing absorption. The triplet He I $\lambda 5876$ line, shown in Fig. 5, displays significant variability, developing a strong P Cygni profile in the 03/04/2004 (MJD = 53 098.4) spectrum and a double-peaked profile separated by $\sim 80 \mathrm{~km} \mathrm{~s}^{-1}$ in the $09 / 06 / 2004(\mathrm{MJD}=53165.2)$ and $10 / 07 / 2004$ $(\mathrm{MJD}=53$ 196.2) spectra. The singlet He I $\lambda \lambda 5015,6678$ lines are also variable, although the double-peaked profile is not seen and the lines instead weaken to barely detectable levels before recovering in the final spectrum.

The lowest He I triplet term, $2 \mathrm{~s}^{3} \mathrm{~S}$, is metastable, and $n\left(2 \mathrm{~s}^{3} \mathrm{~S}\right)$ becomes large as the level is populated by the recombination cascade from $\mathrm{He}^{+}$(Osterbrock \& Ferland 2006). From here, collisional excitation populates the $2 \mathrm{p}^{3} \mathrm{P}^{\circ}$ upper energy level of the He I $\lambda 1.083 \mu \mathrm{m}$ line as well as the higher triplet levels (including the upper levels of He I $\lambda \lambda 5876,7065)$ and the singlet levels responsible for the observed He I $\lambda \lambda 5016,6678$, $2.058 \mu \mathrm{m}$ emission (Bray et al. 2000; Osterbrock \& Ferland $2006)^{7}$. The $2 \mathrm{p}^{3} \mathrm{P}^{\circ}$ level is the lower level of both the He I $\lambda 5876$ and $\lambda 7065$ lines, but the oscillator strength of the He I $\lambda 5876$ line

\footnotetext{
7 Absorption of $\lambda 3889$ photons by the $2 \mathrm{~s}^{3} \mathrm{~S}$ level also populates the $3 \mathrm{p}^{3} \mathrm{P}^{\circ}$ level, with $\sim 10 \%$ of decays proceeding via the $3 \mathrm{~s}^{3} \mathrm{~S}$ level to $2 \mathrm{p}^{3} \mathrm{P}^{\circ}$, strengthening the He I $\lambda 7065$ emission line. van Helden (1972) also proposes that the $3 \mathrm{p}^{3} \mathrm{P}^{\circ}$ level may be populated via O II $\lambda 539.1 \mathrm{flu}-$ orescence from the He I $1 \mathrm{~s}^{1} \mathrm{~S}$ level, which would provide a second mechanism for strengthening the He I $\lambda 7065$ emission.
} 
Table 2. Equivalent widths $(\AA)$ and radial velocities $\left(\mathrm{km} \mathrm{s}^{-1}\right)$ for the $\mathrm{H} \alpha, \mathrm{Pa} \delta$ and $\mathrm{He} \mathrm{I} \lambda 7065$ emission lines ${ }^{a, b}$.

\begin{tabular}{c|ccccc|cc|cc}
\hline \hline & & \multicolumn{3}{|c|}{$\mathrm{H} \alpha$} & \multicolumn{2}{c|}{$\mathrm{Pa} \delta$} & \multicolumn{2}{c}{$\mathrm{He} \mathrm{I} \lambda 7065$} \\
date & $E W$ & $V_{\mathrm{r}}(\mathrm{b})$ & $V_{\mathrm{r}}$ & $V_{\mathrm{r}}(\mathrm{r})$ & $\Delta V_{\mathrm{r}}$ & $E W^{c}$ & $V_{\mathrm{r}}$ & $E W$ & $V_{\mathrm{r}}$ \\
\hline $21 / 09 / 2003$ & $-22.3 \pm 0.2$ & -75.4 & -51.6 & -26.0 & 49.4 & $-1.10 \pm 0.08$ & -52.7 & $-0.79 \pm 0.03$ & -48.9 \\
$03 / 04 / 2004$ & $-25.4 \pm 0.2$ & -71.2 & -58.4 & -32.8 & 38.4 & $-1.13 \pm 0.08$ & -50.2 & $-0.49 \pm 0.05$ & -35.0 \\
$09 / 06 / 2004$ & $-25.2 \pm 0.2$ & -73.8 & -61.4 & -34.0 & 39.8 & $-1.15 \pm 0.08$ & -51.7 & $-0.86 \pm 0.03$ & -45.6 \\
$10 / 07 / 2004$ & $-24.5 \pm 0.2$ & -74.2 & -59.2 & -31.3 & 42.9 & $-1.03 \pm 0.08$ & -50.2 & $-0.87 \pm 0.03$ & -43.3 \\
$28 / 09 / 2004$ & $-23.9 \pm 0.2$ & -72.6 & -61.6 & -31.1 & 41.6 & $-1.05 \pm 0.08$ & -49.3 & $-0.84 \pm 0.03$ & -44.1 \\
\hline $29 / 05 / 2005$ & $-21.2 \pm 0.3$ & - & - & $-34.3 \pm 6$ & - & - & - & $-0.80 \pm 0.06$ & -47.1 \\
\hline
\end{tabular}

${ }^{a}$ For the double-peaked $\mathrm{H} \alpha$ line radial velocities are given for the bluewards and redwards peaks, $V_{\mathrm{r}}(\mathrm{b})$ and $V_{\mathrm{r}}(\mathrm{r})$, the central absorption feature, $V_{\mathrm{r}}$, and the separation of the two peaks, $\Delta V_{\mathrm{r}}$.

${ }^{b}$ Except where noted, errors are conservatively estimated at $\pm 4 \mathrm{~km} \mathrm{~s}^{-1}$ for the $\mathrm{H} \alpha$ radial velocities and $\leq \pm 8 \mathrm{~km} \mathrm{~s}^{-1}$ for the $\mathrm{He} \mathrm{I} \lambda 7065$ and $\mathrm{Pa} \delta$ radial velocities.

${ }^{c}$ The equivalent width of the $\mathrm{Pa} \delta$ line includes the absorption components seen in Fig. 2 on either side of the emission line. If these are excluded, then the equivalent width is broadly constant at $\sim 1.35 \pm 0.15 \AA$ (with increased errors due to the difficulty of estimating the continuum).

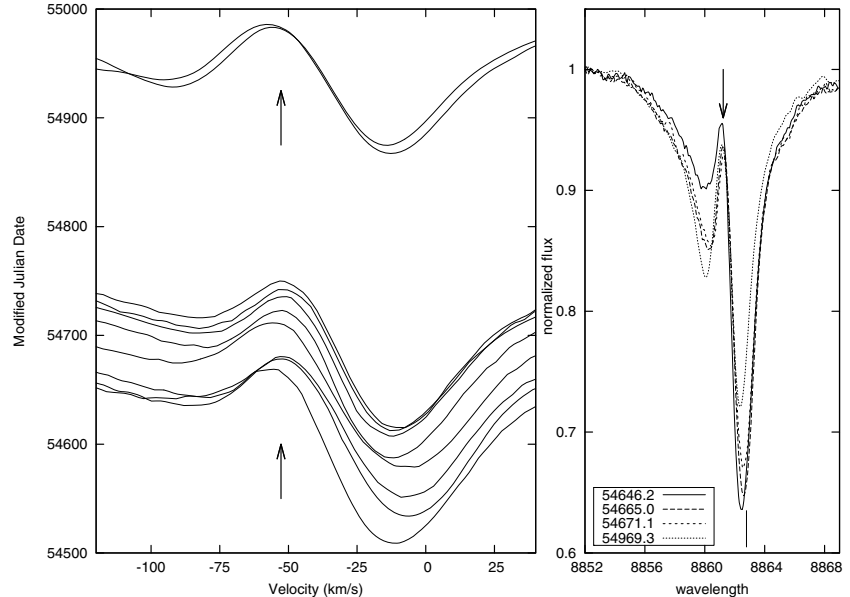

Fig. 3. The Pa11 $\lambda 8863$ feature over the course of the VLT/FLAMES observations. The left panel plots the region around the line centre (with velocities relative to the Pa11 rest wavelength) with spectra offset vertically according to the date of the observation. The right panel overplots four spectra (three from 2008, one from 2009), with the rest wavelength indicated. The radial velocity of the $\mathrm{Pa} \delta$ emission line in the 21/09/2003 (MJD = 52 903.2) VLT/UVES spectrum is marked with an arrow, showing its close correspondence with the emission feature in the Pa11 line and lack of long-term variability.

is almost an order of magnitude greater and absorption is correspondingly more pronounced; this is clearly visible in Fig. 6, which shows the differing absorption bluewards of the two emission lines. Finally, we note that the weak He I $\lambda 8583$ emission arises from a transition from the $10 \mathrm{~d}^{3} \mathrm{D}$ level at $\sim 24.45 \mathrm{eV}$ to the $3 \mathrm{p}^{3} \mathrm{P}^{\circ}$ level (van Helden 1972). Other He I emission lines from the high singlet and triplet levels would be expected, e.g. the related He I $\lambda 8777$ line, but, given their low intensity, detection requires a combination of low-noise spectra and separation from other photospheric, interstellar and telluric features, and none are observed.

\subsubsection{Fe II, Ca II and Mg I emission}

Figure 7 shows the complex Ca II $\lambda 8662 / \mathrm{Pa} 13$ blends over the course of ten VLT/FLAMES spectra of W243. The Ca II emission comes from the multiplet- $24^{2} \mathrm{P}^{\circ}-3^{2} \mathrm{D}$ transition, the upper levels of which are fed by the Calcium- $\mathrm{H}$ and $-\mathrm{K}$ absorption
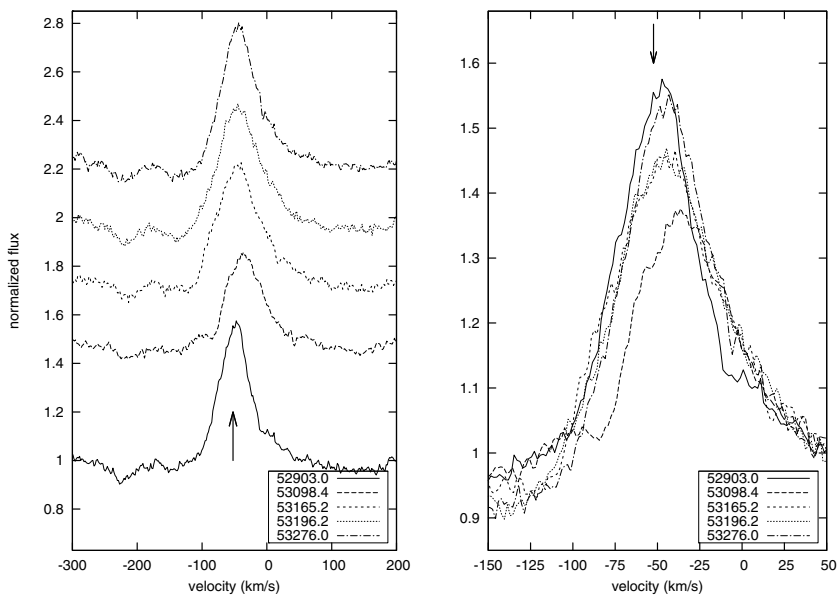

Fig. 4. Evolution of the He I $\lambda 7065$ line over the course of the UVES observations. Spectra are offset vertically in the left panel, and overplotted in the right panel. Velocities are relative to the rest wavelengths of the lines, and the radial velocity of the $\mathrm{Pa} \delta$ line in the 21/09/2003 (MJD = 52 903.2) UVES spectrum is indicated with an arrow.
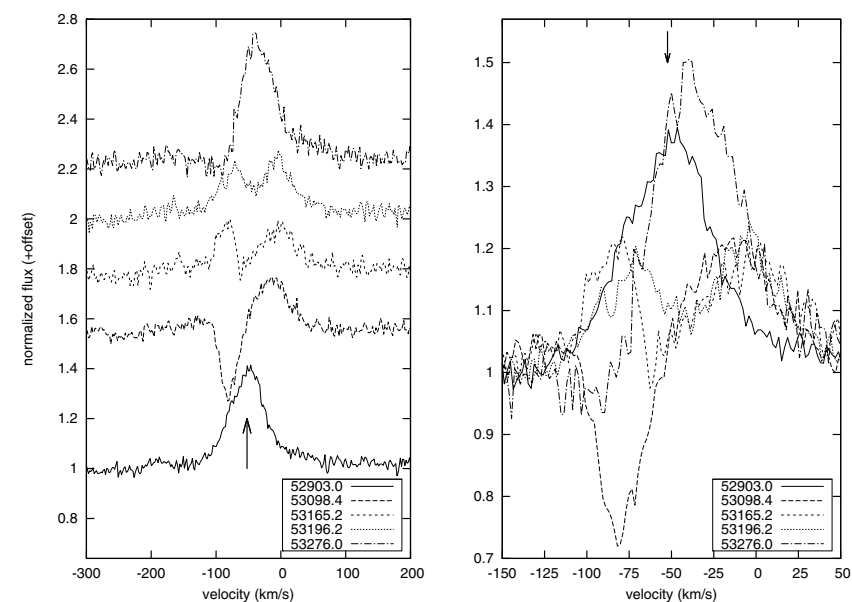

Fig. 5. Evolution of the He I $\lambda 5876$ line over the course of the UVES observations. Spectra are shown in the same manner as Fig. 4.

lines (which at $\lambda \lambda 3933,3968$ are beyond the coverage of our spectra). Unlike the YHG IRC +10 420 (Oudmaijer 1998; Humphreys et al. 2002) we do not observe the accompanying 

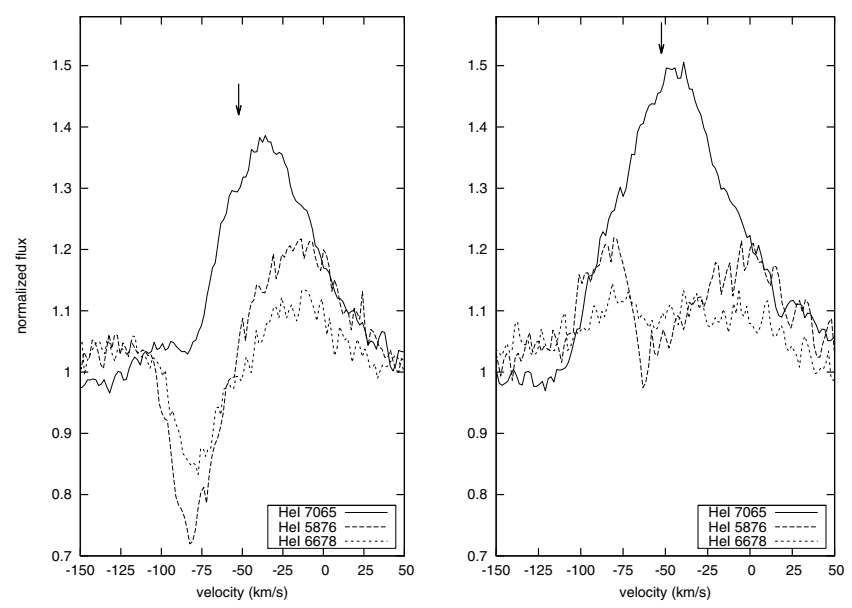

Fig. 6. Comparison of the He I $\lambda \lambda 5876,6678,7065$ emission lines on 03/04/04 (MJD = 53 098.4; left panel) and 09/06/04 (MJD = 53 165.2; right panel). The radial velocity of the $\mathrm{Pa} \delta$ line in the 21/09/2003 (MJD = 52 903.2) UVES spectrum is indicated with an arrow.

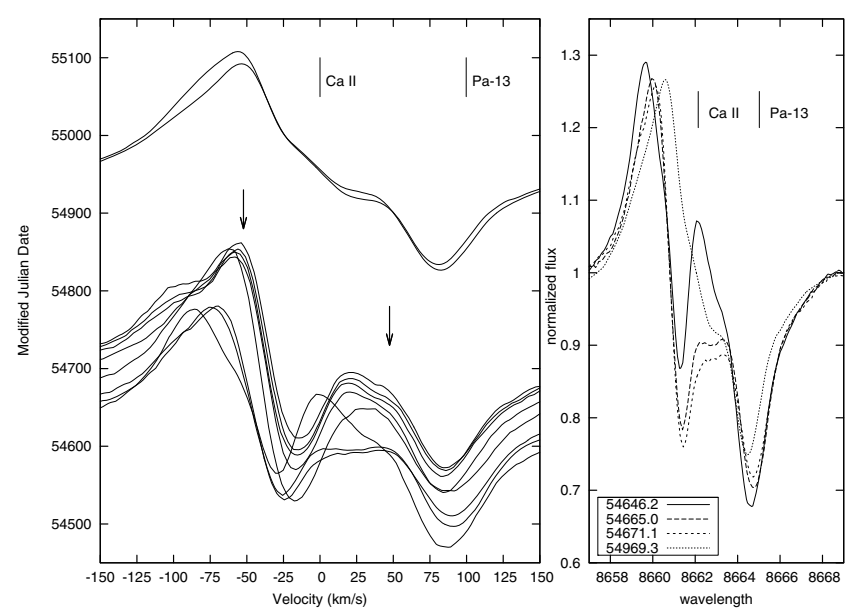

Fig. 7. The evolution of the complex Ca II $\lambda 8662 / \mathrm{Pa} 13$ feaure in the VLT/FLAMES spectra of W243. The left panel plots the region around the Ca II $\lambda 8662$ line (with velocities relative to the rest wavelength), offset vertically according to the date of the observation. The rest wavelength of the Pa13 line is also indicated, and the radial velocity of the $\operatorname{Pa} \delta$ line in the 21/09/2003 (MJD = 52 903.2) UVES spectrum relative to the $\mathrm{Ca}$ II and Pa13 rest wavelengths is indicated with arrows. The right panel overplots four spectra (three from 2008, one from 2009) with rest wavelengths indicated.

[Ca II] $\lambda \lambda 7292,7324$ (multiplet 1F) emission that would result from a subsequent transition to the ground state; this is possibly masked by heavy telluric contamination, but is more probably a result of collisional de-excitation. In the majority of spectra the peak Ca II emission again falls at the same radial velocity as the $\operatorname{Pa} \delta$ and $\mathrm{He}$ I emission, blueshifted with respect to a Ca II absorption feature that is itself heavily blended with the emission component of the adjacent Pa13 line. However, unlike the Paschen-series lines, the Ca II emission extends bluewards $\geq 100 \mathrm{~km} \mathrm{~s}^{-1}$ from the line centre and significant variability can be seen over short timescales in the 2008 dataset, while the central Ca II absorption is entirely absent in the 2009 spectra. Weak Mg I $\lambda 8807$ and very weak Mg I $\lambda 8837$ emission is also seen. These lines arise from upper energy levels at $5.75 \mathrm{eV}$ and $7.52 \mathrm{eV}$, near the ionization potential of $\mathrm{Mg} \mathrm{I}$, and are due to recombination of $\mathrm{Mg}^{+}$and the resultant cascade to the ground

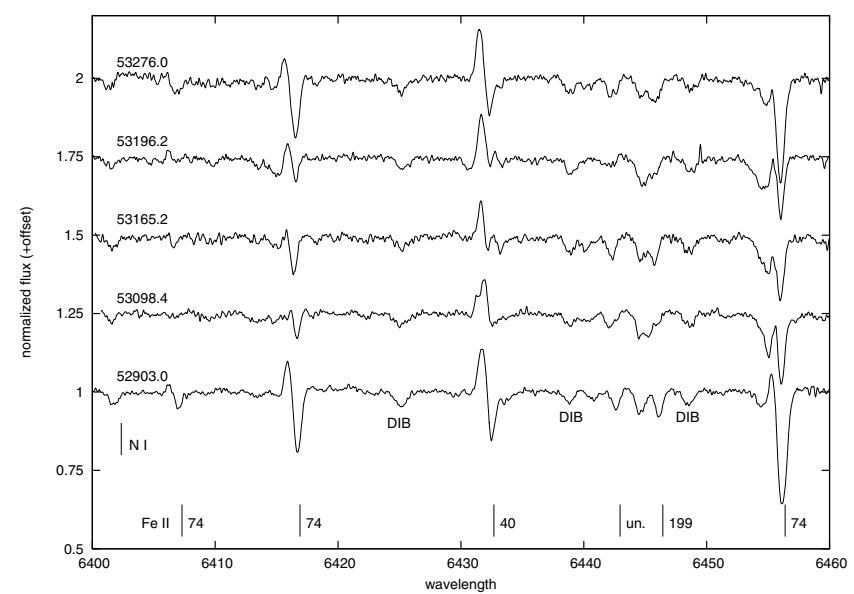

Fig. 8. Evolution of the Fe II multiplet-40 and -74 lines over the course of the UVES spectra. Spectra are labeled with Modified Julian Dates, and the rest wavelengths of the principal Fe II and N I lines are shown.
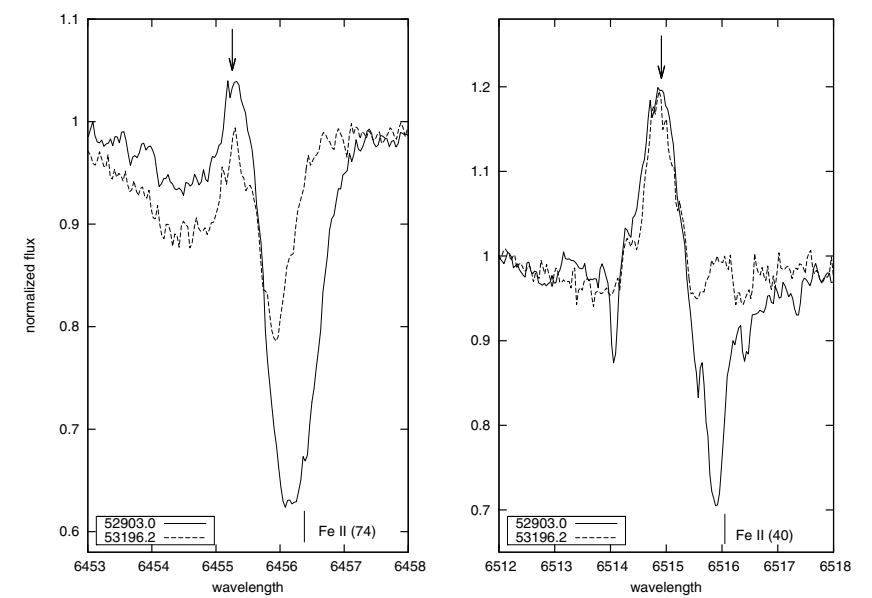

Fig. 9. Comparison of the Fe II $\lambda 6456.4$ (multiplet 74, left panel) and Fe II $\lambda 6516.1$ (multiplet 40, right panel) emission/absorption blends. Two epochs are shown, 21/09/2003 (MJD = 52903.0, solid line) and 10/07/2004 (MJD = 53 196.2, dashed line). Rest wavelengths are marked, and the location of the $\mathrm{Pa} \delta$ line in the 21/09/2003 (MJD = $52903.2)$ spectrum is indicated with arrows. Note the similarity between the behaviour of the Fe II $\lambda 6546.4$ line to the Pa11 line plotted in Fig. 3.

state. Both the $\mathrm{Mg}$ I and Ca II lines are likely to be formed in a neutral $\mathrm{H}$ I region; the ionization potentials of $\mathrm{Ca}$ II $(11.9 \mathrm{eV})$ and $\mathrm{Mg} \mathrm{I}(7.65 \mathrm{eV})$ are too low for significant recombination to take place in an $\mathrm{H}$ II region.

Finally, a large number of Fe II lines are also visible in Fig. 1. The strongest Fe II features are lines from multiplets 40 $\left(a^{6} \mathrm{~S}-z^{6} \mathrm{D}, \chi_{\text {upper }} \sim 4.8 \mathrm{eV}\right)$ and $74\left(b^{4} \mathrm{D}-z^{4} \mathrm{P}, \chi_{\text {upper }} \sim 5.85 \mathrm{eV}\right)$, which display prominent inverse $\mathrm{P}$ Cygni profiles in the initial 21/09/2003 (MJD = 52 903.0) spectrum with emission components again displaying near-identical radial velocities to the other emission lines in the spectrum of W243. These Fe II lines show significant variations in strength and morphology; a number of these lines are plotted in Figs. 8 and 9, which show their evolution over the course of the UVES spectra. The initial inverse P Cygni profiles become less pronounced and the lines of multiplet 40 are seen predominantly in emission, displaying notable $\mathrm{P}$ Cygni profiles in the 10/07/2004 (MJD = 53 196.2) spectrum before the inverse P Cygni profiles reform. In contrast, the multiplet 74 lines are seen mainly in absorption, 

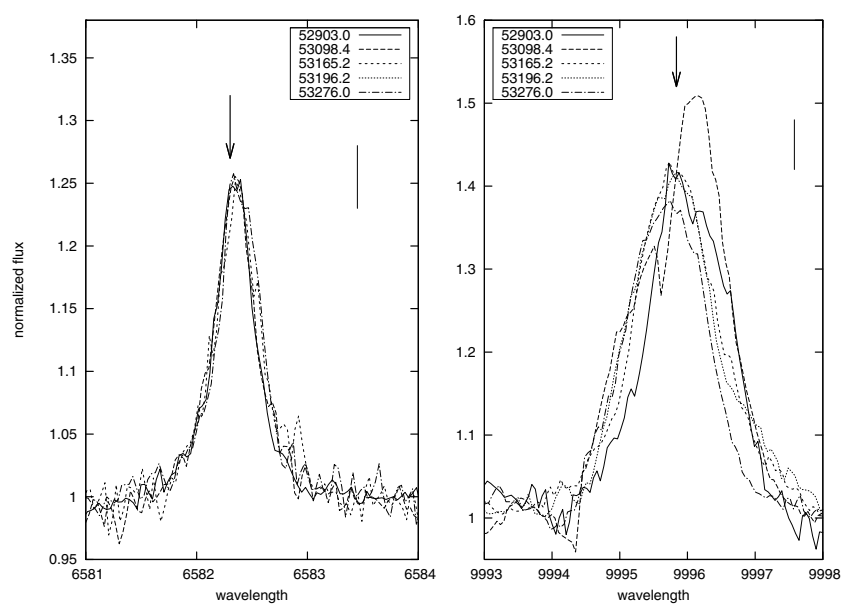

Fig. 10. [N II] $\lambda 6583$ (left panel) and the Ly $\alpha$-pumped FeII $\lambda 9998$ (right panel) emission lines in the UVES spectra of W234. Rest wavelengths are marked, and the location of the $\mathrm{Pa} \delta$ line in the 21/09/2003 (MJD = 52 903.2) spectrum is indicated with an arrow.

with weak emission components on the blue wing that are very similar to the higher Paschen-series lines plotted in Fig. 3. A number of weak Fe II lines display no obvious emission (e.g. Fe II $\lambda \lambda 6175,6332$, multiplets 200 and 199 respectively). These lines all have relatively high upper energy levels, e.g. $x^{4} \mathrm{~F}$ and $x^{4} \mathrm{G}$ at $\sim 8.2 \mathrm{eV}$, multiplets 199 and 200, or the unclassified multiplet containing Fe II $\lambda 6644$ with an upper level at $\sim 9.7 \mathrm{eV}$. At shorter wavelengths there are emission/absorption blends due to Fe II $\lambda \lambda 4924,5018,5169$ (multiplet 42) and Fe II $\lambda \lambda 5235$, 5276, 5316 (multiplet 49), while a large number of weaker Fe II absorption lines below $6000 \AA$ from multiplets 41, 46-48 and 51-57 cannot be examined in detail due to the poor $S / N$ ratio. Notably, no emission or absorption lines of either Fe I or Fe III are observed in the spectra of W243, implying that the iron must be almost entirely in the $\mathrm{Fe}^{+}$state.

\subsection{4. [N II] and [Fe II] emission}

The spectrum of W243 displays [N II] $\lambda \lambda 5754,6548,6583$ forbidden lines (the latter plotted in Fig. 10), with the [N II] $\lambda 6548$ line a factor of $\sim 3$ weaker than [N II] $\lambda 6583$ as expected from the relative radiative transition probabilities. The [N II] lines display virtually unchanging line profiles with a $F W H M \sim 25 \mathrm{~km} \mathrm{~s}^{-1}$ and near-static line centres that are blueshifted by $\sim 50 \mathrm{~km} \mathrm{~s}^{-1}$ from rest wavelengths. [Fe II] $\lambda 7155$ is also seen, while weaker [Fe II] $\lambda \lambda 7388,7453$ lines are observed. At the extreme blue end of the 21/09/2003 (MJD = 52903.0) spectrum possible [Fe II] $\lambda 4799$ emission is detected, but this is not observed in subsequent (lower $S / N$ ratio) spectra. In the first and last UVES spectra the [Fe II] $\lambda 7155$ line appears very similar to the $[\mathrm{N} \mathrm{II}]$ emission with virtually identical $F W H M$ and radial velocities, but the intermediate spectra display more complex, distorted profiles. Very weak [S II] $\lambda \lambda 6716$, 6731 lines are tentatively identified. The [Fe II] $\lambda 7155,7388$, 7453 lines come from the multiplet $14 \mathrm{~F} a^{4} \mathrm{~F}-a^{2} \mathrm{G}$ transition (Moore 1945) with an upper energy level of $1.96 \mathrm{eV}$, and the tentatively-identified [Fe II] $\lambda 4799$ line arises from the multiplet $4 \mathrm{~F} a^{6} \mathrm{D}-b^{4} \mathrm{P}$ transition from a level at $\sim 2.7 \mathrm{eV}$ to the ground state. No higher-excitation lines (e.g. [Fe III], [S III]) are observed, implying a temperature of $\sim 10^{4} \mathrm{~K}$ in the line-forming region (e.g. Lamers 1988).

\subsubsection{Ly $\alpha$ and $\operatorname{Ly} \beta$ fluorescence emission}

In addition to the Fe II lines from multiplets 40 and 74, notable near infra-red emission lines are seen in the 21/09/2003 $(\mathrm{MJD}=52903.0)$ spectrum from Fe II $\lambda \lambda 8451,8490,8927$, 9998 and O I 18446 . These arise from fluorescence by Ly $\alpha$ and $\operatorname{Ly} \beta$ photons (Damineli 2001), which (like the He I emission lines) implies the presence of a hot, ionizing source. The Fe II $\lambda 9998$ line (plotted in the right panel of Fig. 10) is strong in all UVES spectra, displaying moderate variability including a notable strengthening accompanied by a shift redwards in the 03/04/2004 (MJD = 53098.4) spectrum. This line arises from Ly $\alpha$ pumping from the $a^{4} \mathrm{G}$ level (notably the lower level of the strong multiplet 46 and 49 lines seen in the spectrum of W243) to an upper energy level at $\sim 13.4 \mathrm{eV}$, which subsequently feeds the $b^{4} \mathrm{G}$ upper level of the Fe II $\lambda 9998$ line (Johansson 1984). A weaker Fe II $\lambda 9956$ line, produced by the same mechanism, is tentatively identified. Sigut \& Pradhan (1998) predict other fluorescence lines in the 8500-9200 $\AA$ region, resulting from Ly $\alpha$ fluorescence from the $a^{4} \mathrm{D}$ level. These include Fe II $\lambda \lambda 8490,8927$ which are transitions from Ly $\alpha$-pumped ${ }^{4} \mathrm{~F}$ and ${ }^{6} \mathrm{~F}$ levels at $\sim 11.4 \mathrm{eV}$ : both lines are observed in the spectrum of W243. Other predicted lines in the 9100-9200 A range fall in a region of our spectra that is heavily contaminated by telluric features and are not observed. We also observe weak emission from Fe II $\lambda 8451$, which shares a common upper level with Fe II $\lambda 8490$ (Damineli 2001), although this is blended with a telluric absorption line. The majority of the fluorescence lines are only clearly detected in the $21 / 09 / 2003$ (MJD = 52903.0) spectrum, with only Fe II $\lambda 9998$ seen strongly in all UVES spectra and Fe II $\lambda 8927$ seen in the UVES and 2008 FLAMES spectra but absent in the 2009 FLAMES spectra. The other fluorescence lines are either undetectable or, at best, tentatively detectable in the intermediate UVES spectra, and are only weakly observed in the final spectrum.

The O I $\lambda 8446$ line displays an inverse P Cygni profile in the $21 / 09 / 2003$ (MJD $=52903.0$ ) spectrum that is very similar to that seen in the higher Paschen-series and Fe II multiplet-74 lines. The O I $\lambda 7774$ triplet is in absorption and the O I $\lambda 9256$ triplet, although heavily blended with a telluric feature, also appears to be in absorption. This suggests that the O I $\lambda 8446$ emission component is a result of $\mathrm{Ly} \beta$ fluorescence (Bowen 1947); the pumped $3 \mathrm{~d}^{3} \mathrm{D}^{\circ}$ level is linked to the upper level of O I $\lambda 8446$ via the permitted O I $\lambda 11286$ line (outside the coverage of both our spectra and the spectrum of Groh et al. 2007), whereas the $3 d^{3} D^{\circ}$ level is linked with the upper level of the O I $\lambda 7774$ triplet via the [O I] $\lambda 9205$ line, which is not observed. Ionization balance calculations (Grandi 1980) show that $\mathrm{Ly} \beta$ fluorescence of $\mathrm{O}$ I cannot occur in either an $\mathrm{H}$ I region (due to the lack of Ly $\beta$ photons) or an $\mathrm{H}$ II region (as the fluorescence mechanism requires oxygen to be predominantly neutral, and $\mathrm{H} \mathrm{I}$ and $\mathrm{O}$ I have very similar ionization potentials). Instead, the $\mathrm{Ly} \beta$ pumping of O I was found to only be effective in dense, partially-ionized transition zones between $\mathrm{H} \mathrm{I}$ and H II regions (Kwan 1984). The O I 18446 line is not seen in emission or absorption in the spectrum of 03/04/2004 (MJD = 53 098.4), recovering predominantly in absorption in subsequent spectra in a similar manner to the Fe II multiplet 74 lines discussed in Sect. 3.1.3.

Finally, we note that Groh et al. (2006) report Mg II $\lambda 2.14 \mu \mathrm{m}$ emission in their spectrum of $\mathrm{W} 243$. This is another $\mathrm{Ly} \beta$ fluorescence line, with pumping from the $\mathrm{Mg}^{+}$ground state populating the $5 \mathrm{p}^{2} \mathrm{P}^{\circ}$ upper level of the observed $\mathrm{Mg}$ II line (Hamann \& Simon 1988). 

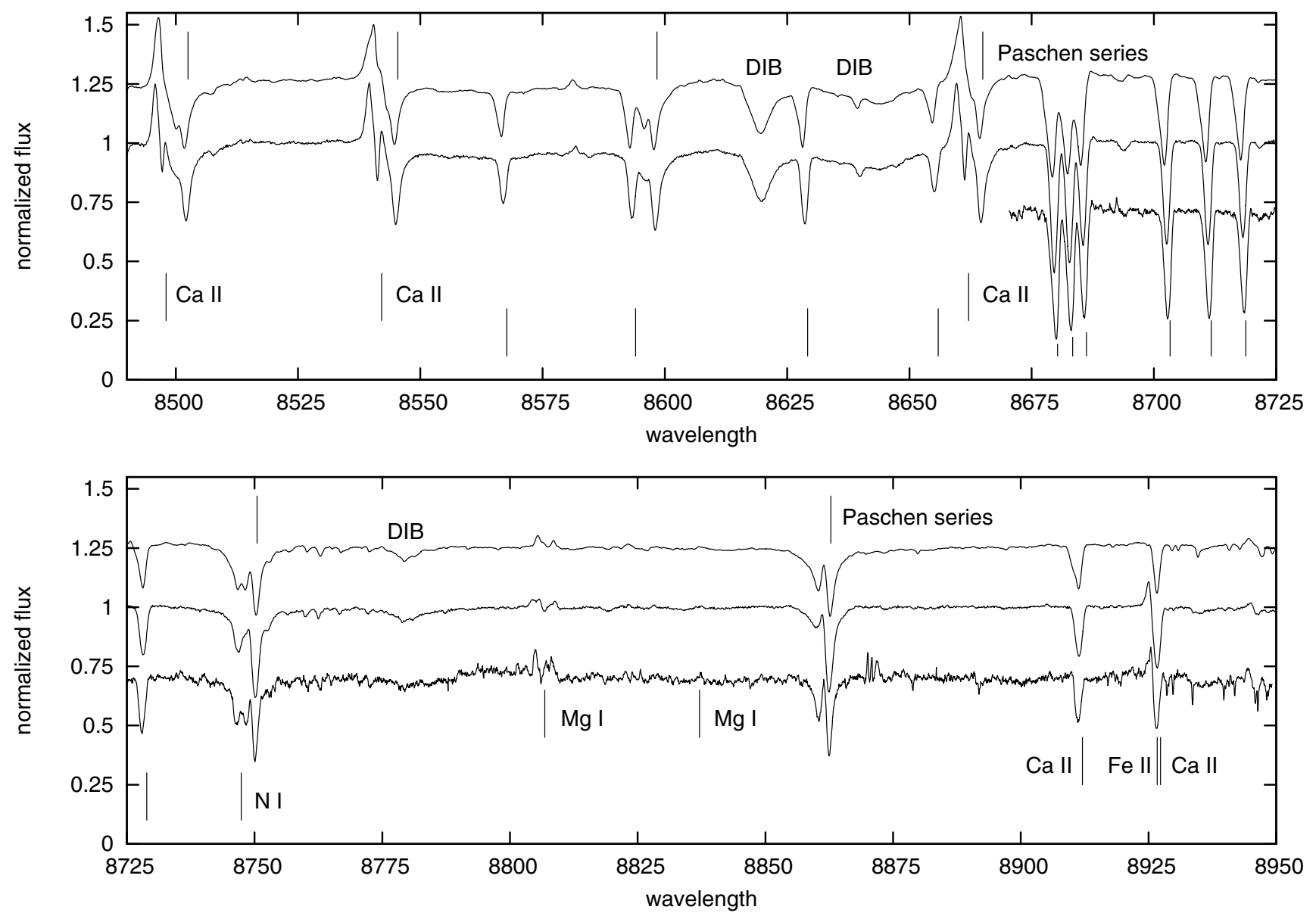

Fig. 11. VLT/FLAMES (top: 18/5/2009, MJD = 54 969.3, middle: 29/6/2008, MJD = 54 646.2) and VLT/UVES (bottom: 28/9/2004, MJD = $53276.0)$ spectra of W243. The rest wavelengths of principal features discussed in the text are indicated, and identified diffuse interstellar bands are also marked.

\subsection{Absorption lines}

\subsubsection{Neutral and singly-ionized metals}

VLT/FLAMES spectra of W243 from 2008 and 2009 covering 8500-8950 ̊ are plotted in Fig. 11, along with a VLT/UVES spectrum from 2004 covering 8670-8950 ̊. Strong N I absorption lines are seen from the $3 \mathrm{~s}^{4} \mathrm{P}-3 \mathrm{p}^{4} \mathrm{D}^{\circ}$ (multiplet 1 ) and $3 \mathrm{~s}^{2} \mathrm{P}-3 \mathrm{p}^{2} \mathrm{P}^{\circ}$ (multiplet 8 ) transitions, along with strong Paschenseries Pa11 ... Pa16 absorption lines. Ca II $\lambda \lambda 8498,8542$, 8662, 8927 display emission/absorption blends, although the Ca II $\lambda 8912$ line is seen in absorption only; this line originates from the same multiplet as Ca II $\lambda 8927$, and it is likely that the emission feature in the latter is a blend with a Fe II $\lambda 8927$ emission line $\left(e^{4} D-5 p^{4} D\right)$ that forms part of a recombination cascade from a Ly $\alpha$-pumped level at $\sim 10 \mathrm{eV}$. Notably, this emission component is absent from the Ca II $\lambda 8927$ line in the 2009 FLAMES spectra.

The strong near-IR N I absorption lines and lack of N II features in the VLT/UVES spectra of W243 imply a spectral type no earlier than A0Ia (Munari \& Tomasella 1999, see also the disappearance of N II in spectra of the LBV HR Car as it cools from $20 \mathrm{kK}$ to $10 \mathrm{kK}$; Nota et al. 1997; and Machado et al. 2002). This is consistent with the strong Si II $\lambda \lambda 6347,6371 \mathrm{ab}-$ sorption lines visible in Fig. 1, which are are a good indicator of temperature for cool stars. These lines are strongest at around $10 \mathrm{kK}$ (i.e. spectral type $\sim \mathrm{A} 0$ ), weakening as the temperature rises (Nota et al. 1997; Davies et al. 2005). Ne I, an additional indicator of tempratures greater than $\sim 10 \mathrm{kK}$, is also absent. Comparison of the N I line strength in the VLT/UVES and VLT/FLAMES spectra in Fig. 11 (along with VLT/FLAMES spectra obtained in 2005 and NTT/EMMI spectra from 2003, neither shown) and Si II line strengths from the VLT/UVES and 2006 NTT/EMMI spectra suggest that W243 has shown little change in state since it finished evolving to the cool phase in 2002-3 (Paper I), although we cannot rule out more significant variability during the periods where we do not have spectroscopic coverage.

\subsubsection{Pulsations}

Figure 12 shows the N I $\lambda 8711$ absorption line from the 2008 and 2009 VLT/FLAMES datasets, with spectra offset vertically according to their acquisition date to highlight changes in the line profiles. The line displays significant variability in strength, profile and line centre, and similar variations are seen in the Pa11 line, plotted in Fig. 3, the Pa13 line visible in Fig. 7 and the strong Si II $\lambda \lambda 6347,6371$ doublet and O I $\lambda 7774$ triplet lines (not shown). Over the course of sixteen epochs of VLT/UVES and VLT/FLAMES data the measured radial velocities from the strong neutral/singly-ionized metal lines lie within a range of $-43 \mathrm{~km} \mathrm{~s}^{-1}--18 \mathrm{~km} \mathrm{~s}^{-1}$ (errors $\leq \pm 3 \mathrm{~km} \mathrm{~s}^{-1}$ ) with individual lines consistent to within a few $\mathrm{km} \mathrm{s}^{-1}$ in each spectrum; only the low-excitation Fe II multiplets displaying P Cygni and inverse $\mathrm{P}$ Cygni profiles in Fig. 8 are discrepant, displaying 


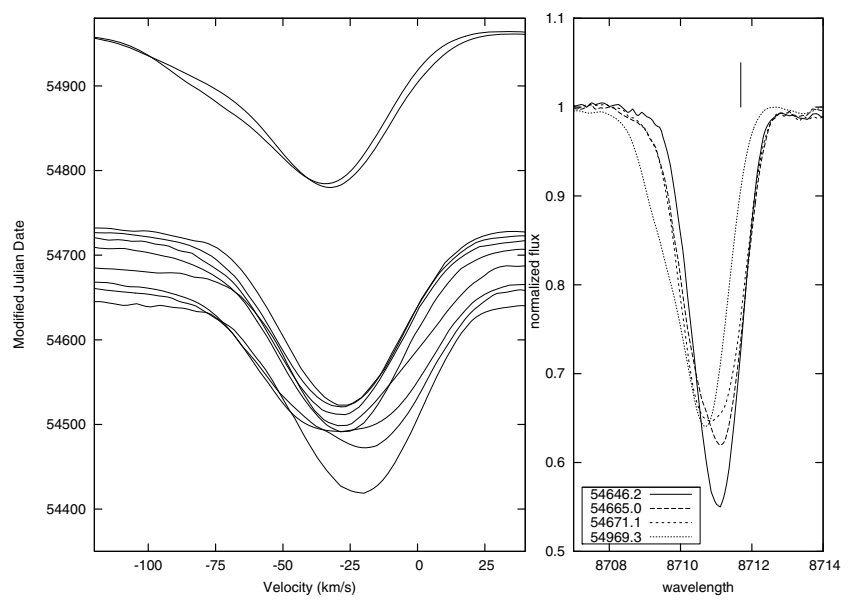

Fig. 12. Evolution of the N I $\lambda 8711$ absorption lines during the VLT/FLAMES observations. As in Figs. 3 and 7, the left panel plots the region around the line centre, with velocities relative to the rest wavelength, for all VLT/FLAMES spectra, offset vertically according to the date of observation. The variability in line centre and profile can be clearly seen, with a broad blue wing extending to $\geq 100 \mathrm{~km} \mathrm{~s}^{-1}$ in the 2009 observations. The right panel overplots four spectra (three from 2008, one from 2009) with the rest wavelength indicated.

red-shifted absorption components relative to the other metal lines, most probably as a result of significant infilling from the blue-wing emission.

As noted in Ritchie et al. (2009), the short-term radial velocity variations are hard to reconcile with orbital motion, as the implied orbital period is at odds with the narrow range of measured radial velocities unless we are viewing the system at $\sin i \sim 0$. In addition, the clear changes in line profile and strength apparent in Fig. 12 are hard to understand in this scenario, and we instead interpret the radial velocity variations as resulting from bulk motions in a pulsating, dynamically-unstable photosphere. Such pulsations are well-documented in YHGs, for example $\rho$ Cassiopaeia (Lobel et al. 1998), and very similar changes in $\mathrm{N} \mathrm{I}$ and Fe I absorption lines are seen in VLT/FLAMES spectra of the pulsating YHG W265 (Ritchie et al. 2009). The limited spectral coverage and lack of contemporaneous photometry makes determination of a pulsation period for W243 impossible; the behaviour of the N I lines in the VLT/FLAMES spectra is consistent with a long pulsational period similar to that observed in YHGs (de Jager 1998), but the photospheric lines also display rapid changes in both radial velocity and profile over a matter of days (e.g. the change between 18/07/2008, MJD = 54665.0 and 24/07/2008, MJD = 54 671.1 visible in Figs. 3 and 12) that could imply that we are observing the effects of sparse sampling of a more rapid pulsational period.

A further similarity with the YHGs $\rho$ Cas and W265 comes from the weakening and broadening of the metal absorption lines through the pulsation period, and in particular the development of the broad blue wing seen in the near-IR N I lines on 10/07/2004 (MJD = 53 196.2, see Fig. 13); the O I and Si II absorption lines display a similar effect. The observed line broadening appears periodic, and is also seen in the $\mathrm{N}$ I lines in the 18/05/2009 (MJD = 54 969.3) VLT/FLAMES spectrum plotted in Fig. 11, most clearly in the N I and Paschen-series absorption lines and the significant reduction in core emission in the $\mathrm{N}$ I triplet at $\sim 8680 \AA$. In $\rho$ Cas this behaviour is correlated with maximum effective temperature (Lobel et al. 1998), and results from absorption in the wind due to a periodic phase of enhanced

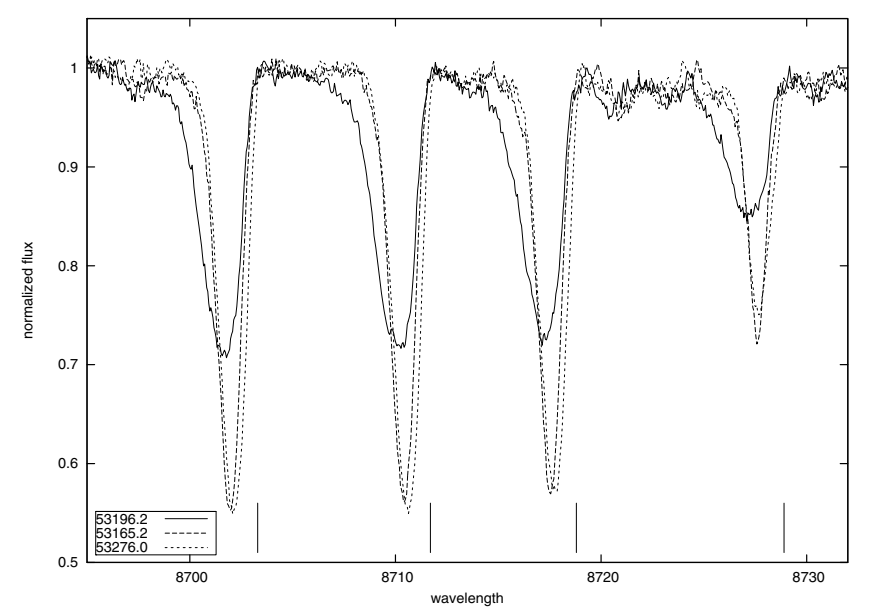

Fig. 13. UVES observations of the N I absorption line complex around $\sim 8700$ A. on 10/07/2004 (MJD = 53 196.2, solid line) with preceeding $(09 / 06 / 2004, \mathrm{MJD}=53$ 165.2) and subseqent $(28 / 09 / 2004, \mathrm{MJD}=$ 53 276.0) spectra plotted with dashed lines for comparison. Rest wavelengths are marked.

mass-loss ${ }^{8}$. W243 therefore appears to be in a relatively quiescent pulsational state similar to warm-phase YHGs, with possible periods of increased mass-loss but no significant changes in state apparent during our monitoring.

\subsubsection{Modeling}

Modeling of W243 was carried out using the non-LTE lineblanketed radiative transfer code CMFGEN (Hillier \& Miller 1998a; Hillier \& Miller 1999), which computes line and continuum formation under the assumptions of spherical symmetry and a steady-state outflow. Examples of the use of CMFGEN to model LBVs and detailed discussion of the workings of the code can be found in Najarro et al. (2009) and Groh et al. (2009) and extensive references therein. For W243, the H/He abundance was fixed at 5, while $\mathrm{C}$ was assumed to be depleted due to the lack of observed lines. $\mathrm{Ti}, \mathrm{Cr}$ and $\mathrm{Ni}$ have been assumed to have solar abundance, whereas abundance determinations for $\mathrm{Fe}, \mathrm{Ca}$, $\mathrm{Mg}, \mathrm{Si}, \mathrm{N}$ and $\mathrm{O}$ are firmly derived. We find that the absorptionline spectrum of W243 is well fit by a cool hypergiant model with a weak, unclumped wind ${ }^{9}$ and low mass-loss rate. Model parameters are given in Table 3; a distance of $4.5 \mathrm{kpc}$ (Clark et al. 2005; Crowther et al. 2006) is assumed, with distance-scaling relationships given as appropriate (Hillier et al. 1998b). The value of $\log g$ is uncertain, with the listed value of $\sim 0.65$ providing the best fit to the relatively uncontaminated higher Paschen-series lines bluewards of $8500 \AA$. Figure 14 shows the model fit to the strong N I multiplet 1 lines and the adjacent Pa12 $\lambda 8750$ line (blended with N I 18747), while Fig. 15 shows the fit to the strong Si II $\lambda 6347,6371$ doublet and O I $\lambda 7774$ triplet lines. All of the strong metal absorption lines are well fit by the model, although the Pa12 line shows significant infilling from the emission component discussed in Sect. 3.1.1. Figure 16 shows three strong Fe II multiplet 40 and 74 lines (two weaker lines from

\footnotetext{
${ }^{8}$ Note that this is not the strongly enhanced mass loss phase encountered by YHGs on timescales of a few decades, accompanied by a significant movement redwards on the HRD (de Jager 1998; Lobel et al. 2003)

9 For the velocity law we assume $V_{\infty}=165 \mathrm{~km} \mathrm{~s}^{-1}$, appropriate for a quiescent YHG, and $\beta=1$.
} 
Table 3. Model parameters for W243.

\begin{tabular}{ll}
\hline \hline Parameter & Value $^{a}$ \\
\hline$L_{*}$ & $7.3 \times 10^{5}(d / 4.5 \mathrm{kpc})^{2} L_{\odot}$ \\
$R_{*}$ & $376.9(d / 4.5 \mathrm{kpc}) R_{\odot}$ \\
$R_{2 / 3}$ & $450.0(d / 4.5 \mathrm{kpc}) R_{\odot}$ \\
$T_{\text {eff }}$ & $\sim 8500 \mathrm{~K}^{b}$ \\
$\dot{M}$ & $6.1 \times 10^{-7}(d / 4.5 \mathrm{kpc})^{1.5} M_{\odot} \mathrm{yr}^{-1}$ \\
$V_{\infty}$ & $165 \mathrm{~km} \mathrm{~s}^{-1}$ \\
$v \sin i$ & $10 \mathrm{~km} \mathrm{~s}^{-1}$ \\
$\log g$ & $\sim 0.65^{c}$ \\
$\mathrm{H} / \mathrm{He}$ & 5 \\
$\mathrm{~N} / \mathrm{N}_{\odot}$ & 12.1 \\
$\mathrm{O} / \mathrm{O}_{\odot}$ & 0.11 \\
$\mathrm{Ca} / \mathrm{Ca} \odot$ & 2.49 \\
$\mathrm{Mg} / \mathrm{Mg} \odot$ & 1.34 \\
$\mathrm{Si} / \mathrm{Si} \dot{ }_{\odot}$ & 1.51 \\
$\mathrm{Fe} / \mathrm{Fe} \odot$ & 1.22 \\
\hline
\end{tabular}

${ }^{a}$ Values for $V_{\infty}, v \sin i$ and $\mathrm{H} / \mathrm{He}$ are assumed, all others are derived. Values are for a distance of $4.5 \mathrm{kpc}$ (Clark et al. 2005; Crowther et al. 2006), with scaling listed where appropriate.

${ }^{b}$ Limits are $8300 \leq T_{\text {eff }} \leq 9300 \mathrm{~K}$, favouring a value at the lower end of this range.

${ }^{c}$ Best fit to the higher Paschen-series absorption lines.

multiplet 199 and a multiplet unclassified by Moore (1945) also visible, along with several weak DIBs), along with the Ca II $\lambda \lambda 8912,8927$ doublet and overlapping Fe II $\lambda 8927$ emission line. In both cases the model reproduces the absorption component well, but does not reproduce the emission components. Similarly, the model predicts Paschen- and Balmer-series lines also in absorption and very weak He I absorption features, rather than the observed emission. Along with the Ly $\alpha$-pumped Fe II and O I emission lines, this implies that the emission-line spectrum does not originate with the cool star but instead has a secondary source; this is discussed further in Sect. 4.

A strong lower limit on temperature come from the Mg I 18807 line, while upper limits come from the He I and $\mathrm{N}$ I lines (the strength of the latter also used as a temperature diagnostic in Paper I). Precise temperature determination is complicated by the presence of emission components in both He I and $\mathrm{Mg} \mathrm{I}$, and we find $8300 \leq T_{\text {eff }} \leq 9300 \mathrm{~K}$, (i.e. spectral type $\left.\mathrm{A} 2-4 \mathrm{Ia}^{+}\right)$, favouring a value of $\sim 8500 \mathrm{~K}\left(\mathrm{~A} 3 \mathrm{Ia}^{+}\right)$. At lower temperatures the $\mathrm{Mg}$ I absorption component would become too strong, even considering infilling from the superimposed emission visible in Fig. 11, while at higher temperatures the unblended N I absorption lines become too weak. $\mathrm{N}$ is highly overabundant $(12.1 \times$ solar $)$, with $\mathrm{O}$ depleted $(0.11 \times$ solar $)$ and $\mathrm{C}$ highly depleted. This is clearly indicative of CNO-processed material being present at the surface of W243, with abundances similar to those determined for AG Car (Groh et al. 2009). Ca is also notably overabundant, with $\mathrm{Mg}, \mathrm{Si}$ and $\mathrm{Fe}$ all moderately enhanced relative to solar values.

\section{Discussion and conclusions}

\subsection{The spectrum of W243}

We summarize the spectrum of W243 as follows:

- H, He I, O I, Mg I, Ca II, Fe II, [N II] and [Fe II] are seen in emission. Strong $\mathrm{H} \alpha, \mathrm{H} \beta$ and $\mathrm{Pa} \delta$ emission is seen,

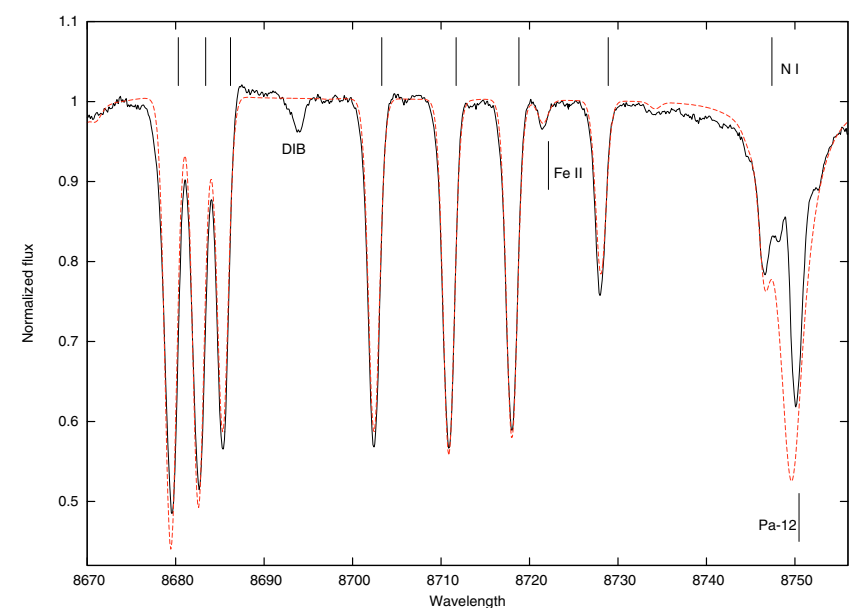

Fig. 14. Comparison of the model fits to the strong N I multiplet 1 absorption lines and adjacent Pa11 $\lambda 8750$ line. The VLT/FLAMES spectrum is from $04 / 09 / 2008$ (MJD $=54713.0)$ and rest wavelengths are marked.

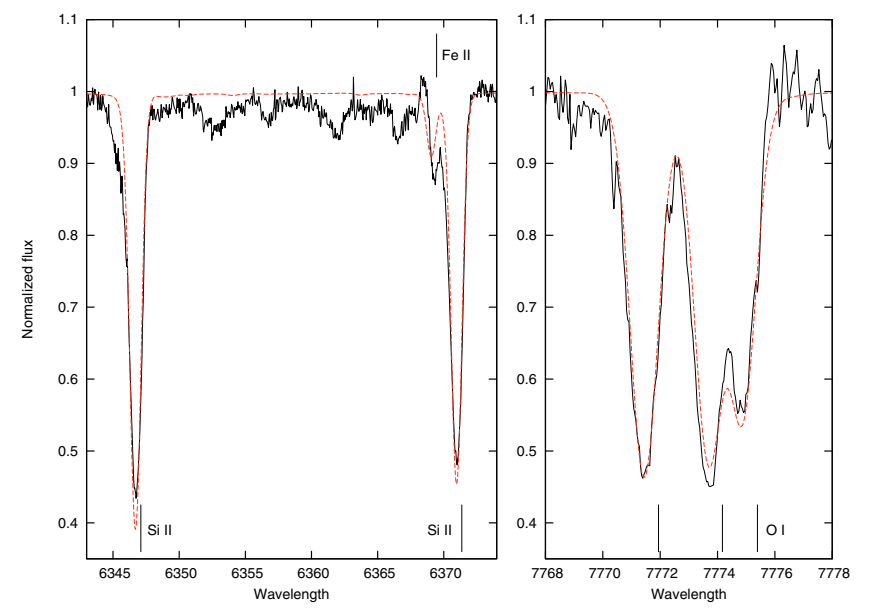

Fig. 15. Model fits to the Si II $\lambda \lambda 6347,6371$ doublet (left panel) and O I $\lambda 7774$ triplet (right panel). The VLT/UVES spectrum is from 21/09/2003 (MJD = 52 903.0) and rest wavelengths are marked.

with the $\mathrm{H} \alpha$ and $\mathrm{H} \beta$ lines displaying double-peaked profiles, while the higher Paschen-series lines show core emission on the blue absorption wing. He I emission is highly variable, while O I $\lambda 8446$ and many Fe II lines also show significant variability. [N II] and a few [Fe II] lines are observed. Apart from a few highly-distorted lines, all permitted and forbidden emission lines display a blueshift of $\sim 50 \mathrm{~km} \mathrm{~s}^{-1}$ with negligible epoch-to-epoch variability.

- Many neutral or singly-ionized metals are seen in absorption. Strong absorption lines of Si II, N I and O I are seen, along with many Fe II lines; Fe I and Fe III features are not observed. Many weaker lines from singly-ionized iron-group elements are also seen. Modeling of the absorption line spectrum shows $8300 \leq T_{\text {eff }} \leq 9300 \mathrm{~K}\left(\mathrm{~A} 2-\mathrm{A}_{4} \mathrm{Ia}^{+}\right.$, with $T_{\text {eff }} \sim$ $8500 \mathrm{~K}$ preferred). This is strongly inconsistent with the observed He I emission and Ly $\alpha$ and $\operatorname{Ly} \beta$ fluorescence lines of $\mathrm{O}$ I and Fe II, implying the presence of an unseen source of ionizing photons.

- Photospheric pulsations are apparent in the metal absorption lines, with periodic broadening and the development of excess blue-wing absorption suggestive of periods 

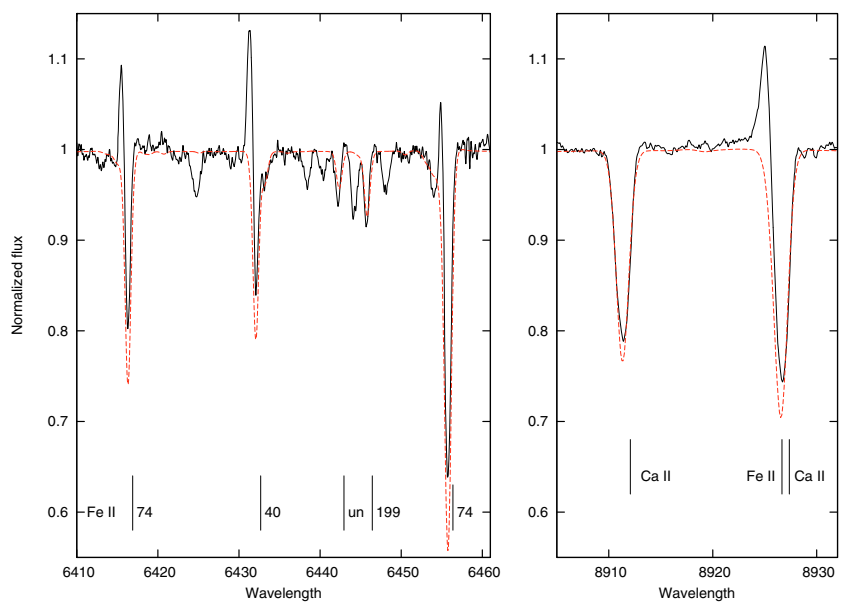

Fig. 16. Example of model fits to emission/absorption blends. The left panel shows three strong Fe II multiplet 40 and 74 lines, while the right panel shows the Ca II $\lambda \lambda 8912,8927$ doublet and superimposed Fe II $\lambda 8927$ emission line. In both cases the absorption component is well fit by the model, but the cool star cannot reproduce the emission component. The VLT/UVES spectrum is from 21/09/2003 (MJD = 52 903.0) and the VLT/FLAMES spectrum is from 04/09/2008 (MJD = 54 713.0). Rest wavelengths are marked, and the Fe II multiplets are also indicated in the left panel.

of increased mass loss. However, no evidence is seen for eruptive mass loss, and W243 appears to be in a quiescent cool-phase state.

\subsection{The current state of W243}

With $L_{*}=7.3 \times 10^{5} L_{\odot}$ (assuming $d=4.5 \mathrm{kpc}$; Clark et al. 2005) and $T_{\text {eff }} \sim 8500 \mathrm{~K}$, W243 lies near the post-Main Sequence track of a $\sim 40 M_{\odot}$ star (e.g. Schaller et al. 1992), falling as expected between the $\sim 40-55 M_{\odot}$ progenitors of the $\mathrm{Wd} 1$ Wolf-Rayet population (Crowther et al. 2006) and the $\leq 30 M_{\odot} \sim$ O7-8V stars at the main sequence turn-off (Negueruela et al. 2009, in prep.). W243 appeared on the blue side of the HRD as a luminous, early-B supergiant when observed by Borgman et al. (1970), Lockwood (1974) and Westerlund (1987) before undergoing an (unobserved) event that led to W243 moving redwards to the "yellow void" and taking on its current appearance as an early-A hypergiant. The final stages of this evolution were seen in the early observations described in Paper I, with the star remaining generally quiescent in its cool-phase state since 2003. Our modeling shows nitrogen to be highly overabundant, with the depletion of carbon and oxygen suggesting we are observing significant quantities of CNO-processed material. Other $\alpha$-process elements $(\mathrm{Ca}, \mathrm{Mg}, \mathrm{Si})$ are also enhanced. The implication is therefore that W243 is either in an advanced pre-RSG LBV phase, having already ejected its less processed, Hydrogenrich outer layers, or has evolved through the RSG phase and returned to the blue side of the HRD, with the current CNO-rich material dredged up during the RSG phase. W243 does not display the extended circumstellar ejecta expected for either evolutionary stage, but, as commented on in Paper I, this is likely to be rapidly dispersed in the extreme environment of $\mathrm{Wd} 1$. While the N/O ratio in W243 is similar to AG Car (Groh et al. 2009), the latter object is both more massive and (considerably) more luminous than W243 and the same evolutionary phase cannot be assumed, while post-RSG objects such as IRC +10420 are also overabundant in Nitrogen (Oudmaijer et al. 2009) ${ }^{10}$. However, future abundance studies of the unique coeval population of early-B supergiants, YHGs and $\mathrm{BIa}^{+} / \mathrm{WNVL}$ Wolf-Rayet precursors found in Wd1 offer the opportunity to accurately constrain both the evolutionary state of W243 itself and the general passage of massive stars as they leave the main sequence and evolve through the "zoo" of transitional objects towards the Wolf-Rayet phase.

\subsection{Comparison with other stars}

\subsubsection{Cool-phase LBVs}

The visual and near-IR spectrum of W243 shows many similarities with other cool-phase LBVs. The Si II doublet is prominent in cool supergiants (Davies et al. 2005), and is therefore reported in many objects, while the strong near-IR N I lines are also seen at $\sim$ B8Ia and later, e.g. in the spectra of S Dor (Munari et al. 2009) and HR Car ${ }^{11}$ (Machado et al. 2002). The $R$-band spectrum of W243 shows strong similarities with the A2 $\mathrm{Ia}^{+} \mathrm{LBV}$ HD 160529 (Stahl et al. 2003; Chentsov et al. 2003), although subtle differences are present, with the C II $\lambda \lambda 6578,6583$ doublet notable in the spectrum of HD 160529 and Ne I also reported; neither can be robustly identified in the case of W243. The rich Fe II spectrum is also a typical feature of cool-phase LBVs, with HR Car a canonical example, displaying prominent Fe II emission/absorption blends in the cool states of 1991 and 1998-2002 that fade as the LBV moves bluewards on the HRD. The transition from P Cygni to inverse P Cygni profiles in Fe II seen in Fig. 8 is also reported in the cases of S Dor (Wolf \& Stahl 1990; Wolf 1992), R127 (Wolf 1992; Walborn et al. 2008) and HR Car (Nota et al. 1997; Machado et al. 2002; Crowther, priv. comm. 2009); we return to this issue in Sect. 4.5.

However, notable differences also exist between W243 and other early-A LBVs. The $\mathrm{H} \alpha$ and $\mathrm{H} \beta$ lines seen in the spectrum of W243 are unusual, appearing strongly in emission but lacking the P Cygni absorption component generally seen in LBV spectra. The strong He I and Ly $\alpha$-pumped Fe II and O I emission in W243 are also clearly discrepant with the cool-phase emission and absorption lines and are more in keeping with early-B/Ofpe hot-phase LBVs (e.g. AG Car; Groh et al. 2009), although other characteristic hot-phase features such as emission lines of Fe III, N II, Si II and Si III are absent. The He I $\lambda \lambda 5876,6678$ lines are seen in absorption in the cool-phase spectra of HR Car (Machado et al. 2002), and the four B6-A2 (candidate) LBVs described by Chentsov et al. (2003), while He I lines appear in emission in R127 only as the Fe II spectrum fades during the transition to the hot, quiescent state (Walborn et al. 2008). HD 160529 provides a striking example of these differences, with the otherwise-similar A2 $\mathrm{Ia}^{+}$spectrum showing $\mathrm{He} \mathrm{I}$ in absorption and a strong P Cygni profile in the $\mathrm{H} \alpha$ and $\mathrm{H} \beta$ lines; our model also suggests that $\mathrm{He} \mathrm{I}$ should be visible weakly in absorption, rather than the observed strong emission. Infra-red spectra of the mid-B or later (candidate) LBVs listed by Groh et al. (2007) again show W243 to be atypical, with a strong He I $\lambda 1.083 \mu \mathrm{m}$ emission line (of slightly greater strength than the adjacent Pa $\gamma$ line) contrasting with the

\footnotetext{
${ }_{10}$ Sodium abundances are used to infer a post-RSG phase for $\rho$ Cas (e.g. de Jager \& Nieuwenhuijzen 1997), but the only features apparent in our spectra result from interstellar absorption.

11 The N I lines are notably stronger in W243 than in HR Car, implying a later spectral type (Munari \& Tomasella 1999).
} 
remainder of the sample that show the line to be in absorption in two cases (HD 168625, HD 168607), almost absent in HR Car and showing a weak emission component with strong P Cygni absorption in HD 160529.

\subsubsection{Yellow hypergiants}

Our modeling shows that the absorption line spectrum of W243 is well described by a cool hypergiant close to the Eddington limit, and we observe spectral variability that is similar to the well-studied YHGs $\rho$ Cas (Lobel et al. 1998) and HR 8752 (de Jager \& Nieuwenhuijzen 1997). Within Wd1, W265 also displays pulsational variability in the near-IR N I lines, while at radio wavelengths $\mathrm{W} 243$ is very similar to the F2 $\mathrm{Ia}^{+}$YHG W4 (Dougherty et al. 2009). The lack of Fe I features indicates that W243 is hotter than these objects, which show strong neutral metal absorption and a complete absence of He I, with the latter also not observed in the A-type YHGs W12a $\left(\mathrm{A} \mathrm{Ia}^{+}\right)$and W16a $\left(\mathrm{A}_{2} \mathrm{Ia}^{+}\right)$. The peculiar mid-A YHG IRC +10 420 (Oudmaijer 1998; Humphreys et al. 2002) shows the strongest spectral similarities to W243, with Fe II multiplet 40 and 74 lines in emission and a strong, double-peaked $\mathrm{H} \alpha$ emission line with broad electron-scattering wings; both objects also display double-peaked Ca II emission. However, the close agreement between the $\mathrm{H} \alpha$ and Ca II features in velocity space (e.g. Fig. 5, Humphreys et al. 2002) is not seen in W243 ${ }^{12}$, suggesting that the origin may not be the same. In addition, the 2009 VLT/FLAMES spectra show that the Ca II infra-red triplet no longer displays a double-peaked profile, with only a weak "step" in the red flank of the emission line hinting at the previous location of the absorption feature. Lack of contemporaneous $R$-band spectra means that we cannot tell if the doublepeaked $\mathrm{H} \alpha$ profile is also now absent, although we note the clear weakening in the redwards peak in the VLT/UVES spectra and its apparent absence in the lower-resolution VLT/FLAMES LR6 spectra from 2005 (see Fig. 2). Therefore, although the doublepeaked line profiles seen in W243 may correspond to the disk (e.g. Jones et al. 1993) or bipolar outflow (Oudmaijer et al. 1994; Davies et al. 2007) models proposed for IRC $+10420^{13}$, the central absorption may also result from radiative transfer effects in the line-forming region; additional modeling would be required to confirm this.

\subsection{Origin of the emission line spectrum}

While the spectrum of W243 shows similarities with both cool, A-type LBVs and A- and F-type YHGs, the juxtaposition of a cool hypergiant absorption line spectrum with hot-phase LBV $\mathrm{He}$ I and Lyman-pumped Fe II and O I emission lines is an obvious discrepancy. However, our model is unable to reproduce any of the observed emission features while simultaneously preserving the N I and Si II absorption lines, and we interpret this as an indication that W243 is a binary, consisting of the coolphase LBV and an undetected hot OB (or possibly Wolf-Rayet) companion that is the source of the ionizing flux responsible

\footnotetext{
12 This may be a result of the blending with the adjacent Paschenseries absorption/emission features which are not seen strongly in IRC +10420 .

13 Humphreys et al. (2002) also propose a complex rain model for IRC +10420 in which both inflow and outflow are present in an opaque wind. However, this requires a mass-loss rate $\sim 10^{3}$ times greater than currently appears to be the case for W243.
}

for the observed radio emission and discrepant emission lines. The binary fraction amongst massive stars in $\mathrm{Wd} 1$ is known to be large (Crowther et al. 2006; Clark et al. 2008; Ritchie et al. 2009), and although W243 lacks the strong non-thermal radio and X-ray emission that provide evidence for colliding winds in a binary system, weak X-ray emission is observed (Clark et al. 2008). Despite the implied presence of a hot companion to the $\mathrm{LBV}$, radial velocity measurements are not obviously consistent with binarity unless we are viewing W243 at a highly favourable angle or the companion is in a long-period, possibly highlyeccentric orbit around the LBV primary as has been suggested for $\eta$ Carinae (Nielsen et al. 2007). An alternative configuration may be similar to that observed in the YHG HR 8752, where the flux from a B1V companion in a wide orbit is responsible for the formation of the observed [N II] emission and a compact radio nebula, neither of which could be formed by the cool hypergiant itself (Stickland \& Harmer 1978). A similar scenario may also apply to the YHG W265, which also displays [N II] and radio emission (Ritchie et al. 2009). In the case of W243 a hotter companion is probably required to provide the ionizing flux necessary to form the observed He I and Ly $\alpha$-pumped emission, both absent in HR 8752 and W265, although we note the absence of higher-excitation emission lines such as N II, [S III], [Fe III] and [Ar III] that are observed in the sgB[e] binary W9 (Clark et al. 2005, 2008), which also displays far stronger Ly $\alpha$-pumped lines than those observed in W243.

The lack of observed Fe I or Fe III features in the spectrum of W243 is unusual and implies that almost all of the iron is in the $\mathrm{Fe}^{+}$state in the line formation region. This is reminiscent of the B and D Weigelt blobs of $\eta$ Carinae (Verner et al. 2002), which display spectra dominated by Fe II and [Fe II] emission originating from upper levels around $6 \mathrm{eV}$ (permitted lines) and $2 \mathrm{eV}$ (forbidden lines). This Fe II spectrum arises from collisional excitation at $10^{6}<n_{\mathrm{e}}<10^{7} \mathrm{~cm}^{-3}$ populating the lower Fe II levels, with strong continuum pumping routes to $\sim 6 \mathrm{eV}$ levels arising from the $a^{4} \mathrm{D}$ and $a^{2} \mathrm{G}$ levels (the $a^{2} \mathrm{G}$ level is the upper level of our observed [Fe II] multiplet $14 \mathrm{~F}$ emission) and Ly $\alpha$ pumping from the $a^{4} \mathrm{D}$ and $a^{4} \mathrm{G}$ levels; this also appears a plausible model for the Fe II emission region of W243 ${ }^{14}$. The significant variability in Fe II, He I and O I emission suggests that the efficiency of the pumping channels varies, possibly due to changes in Lyman-series optical depth or other radiative transfer effects.

\subsection{Location of the emission line formation region}

The transition from P Cygni to inverse P Cygni profiles in the Fe II lines seen in Fig. 8 is observed in other LBVs, and is interpreted as an indication of infall during the radial contraction phase of the stellar photosphere during the S Dor variability cycle (Wolf \& Stahl 1990). In the case of W243, this transition occurs at a time when the strong Si II and N I absorption lines develop broad blue wings ${ }^{15}$ but inspection of the Fe II multiplet 74 and higher Paschen-series lines suggests that these may not be

\footnotetext{
14 The similarity between the Fe II-rich spectra of W243 and other coolphase LBVs, e.g. HR Car or S Dor, also suggests similar conditions in the Fe II line formation regions, although we do not suggest that a hot companion is required in all of these objects; instead, the similarity probably reflects the complex balance of collisional, continuum and Ly $\alpha$-pumping processes in these objects (Verner et al. 2002). 15 A similar transformation is correlated with periods of enhanced mass-loss in the YHG $\rho$ Cas (Lobel et al. 2003).
} 
true (inverse) P Cygni profiles, and rather an effect of emission at near-constant radial velocity superimposed on an absorption line with a varying line centre: this is similar, but more pronounced, to the effects seen in $\rho$ Cas where static emission lines and variable absorption lines produce apparent line-splitting (see Lobel et al. 1998, Figs. 13 and 15). With W243, if the absorption is at its most blueshifted (and especially if a broad blue wing also develops) the emission/absorption blend will take on an apparent $\mathrm{P}$ Cygni profile (e.g. as visible in the $\mathrm{Pa} \delta$ line in the left panel of Fig. 2 in the 10/07/2004, MJD = 53196.2 spectrum, or in the Fe II multiplet 40 lines in Fig. 8), while at its most redshifted the absorption component will be seen entirely on the red flank of the emission component, creating an inverse P Cygni profile: however, neither case corresponds to the significant outflow or inflow normally associated with these spectral features.

The near-static radial velocities of the undistorted emission lines over the course of our observations and the close correspondence between the radial velocities of the permitted and forbidden lines suggests that they all form at significant radii, when material expanding slowly at constant velocity is ionized by the flux from the hot secondary. As an emission spectrum was not observed by Westerlund (1987), it appears that this material is a relic of mass-loss during the transition from an earlyB supergiant to the current cool state. The O I 18446, Fe II and higher Paschen-series lines have very similar profiles, and all likely form in a common region in the boundary zone between $\mathrm{H}$ I and $\mathrm{H}$ II regions required for efficient $\mathrm{Ly} \beta$-pumping of the O I line, while the consistency between the measured radial velocities of almost all the permitted and forbidden emission lines suggests formation in the same comoving region. The radial velocity of the $\mathrm{Ca}$ II emission is somewhat variable, while the line is broader than all but the Balmer-series emission lines, most probably reflecting formation in a different location: the $\sim 11.9 \mathrm{eV}$ ionization potential means recombination will be negligible in a $\mathrm{H}$ II region, and the line likely results from resonance absorption in an $\mathrm{H}$ I region rather than being limited to the $\mathrm{H} \mathrm{I} / \mathrm{H}$ II transition zone. It is plausible that the Ca II emission may be a result of dust evaporation (e.g. Prieto et al. 2008) which would indicate a post-RSG state for W243, although the [Ca II] emission that would provide stronger support for this hypothesis is absent (Shields et al. 1999). These lines lie in a region of heavy telluric contamination, but we also note that [Ca II] is only weakly detected in the B[e] star MWC 349A despite strong Ca II emission being present (Andrillat et al. 1996). The inferred electron density in the line formation region is high (Hamann \& Simon 1988), and it is likely that the Ca II $3^{2} \mathrm{D}$ level is collisionally de-excited before the forbidden transition can occur. This also appears likely in the case of W243, where relatively strong [Ca II] emission would be required to be detectable amidst the telluric lines.

\subsection{Conclusions and future work}

Our observations of W243 show that it has remained in a quiescent, cool-phase state since 2003, with no indication of either further movement redwards or a return to its former hot state. The $\sim \mathrm{A}_{3} \mathrm{Ia}^{+}$absorption-line spectrum displays photospheric pulsations and episodic mass loss that appears similar to warm YHGs (e.g. $\rho$ Cas, Lobel et al. 1998 or W265; Ritchie et al. 2009). The high Nitrogen abundance and depletion of Oxygen and Carbon imply that CNO-processed material is on the surface of W243, indicating an advanced evolutionary state and significant previous mass-loss. Superimposed on the YHG absorption-line spectrum are emission lines of H, He I, Ca II, Fe II and $\mathrm{O}$ I formed at large radii when material lost from the LBV is ionized by an unseen hot companion. We are unable to strongly constrain the emission-line formation region and the origin of the peculiar, double-peaked Balmer-series profiles or the highly variable He I emission lines. However, highresolution infra-red spectroscopy with adaptive optics offers the opportunity to directly probe the line-forming region and thereby determine the recent mass-loss history of W243. In addition, abundance studies of the coeval population of both pre- and postLBV transitional stars in Wd1 will allow the evolutionary state of both W243 and its evolutionary contemporaries to be determined.

Acknowledgements. J.S.C. gratefully acknowledges the support of an RCUK fellowship. This research is partially funded by grants AYA2008-06166-C0302, AYA2008-06166-C03-03 and Consolider-GTC CSD-2006-00070 from the Spanish Ministerio de Ciencia e Innovación (MICINN). We thank the referee, Otmar Stahl, for valuable comments.

\section{Appendix A: List of identified absorption lines in the spectrum of W243}

W243 displays a large number of absorption lines from neutral and singly-ionized metals; these are listed by species and multiplet in Table A.1. To identify the lines we followed the approach of Oudmaijer (1998), first identifying strong absorption lines and then searching for other lines in the same multiplet using the line identification tables of Moore (1945). We then searched for other multiplets of the same species, and regard as robust any identification where several lines from the same multiplet are unambiguously detected. In a few cases tentative identifications are made when only one line in a given multiplet is detected, provided that one of the following conditions apply:

- the multiplet contains no other lines, or the other expected lines in the multiplet are outside the range of coverage of the spectrum;

- the proposed identification is from a species that has been identified in other multiplets, the line is not close to possible interstellar or telluric features and no other plausible identification exists;

- the proposed identification is from an otherwise unidentified species, but the line is close to a robustly identified line (allowing the difference between line centres $\delta \lambda=\lambda_{\text {known }}-$ $\lambda_{\text {unknown }}$ to be measured accurately) and no other plausible identification exists with the measured $\delta \lambda$. Nevertheless, such an identification should be considered tentative.

The majority of absorption lines in the range 4800 to $\sim 7000 \AA$ could be positively identified in this manner. After eliminating obvious telluric features, many remaining absorption features could be identified as being due to diffuse interstellar bands by comparison with the Diffuse Interstellar Band Catalog ${ }^{16}$, which is based on Jenniskens \& Desert (1994). The large number of telluric features above $7000 \AA$ made comprehensive identification in that region problematic, and our list is incomplete in that wavelength range with only the stronger features being identified.

${ }^{16}$ http://leonid.arc.nasa.gov/DIBcatalog.html 
Table A.1. Identified absorption lines in the spectrum of W243 between 4800-9000 A.

\begin{tabular}{|c|c|c|}
\hline & Multiplet $^{\dagger}$ & Rest wavelength $(\AA)$ \\
\hline Ca II & $\begin{array}{c}2 \\
\text { uncl. }\end{array}$ & $\begin{array}{l}8498.02^{a}, 8542.09^{a}, 8662.14^{a} \\
8912.07,8927.36^{a}\end{array}$ \\
\hline Cr II & $\begin{array}{c}24 \\
29 \\
30 \\
43 \\
50 \\
105\end{array}$ & $\begin{array}{l}5153.49,5305.85 \\
5369.25,5395.41,5409.28,5430.41 \\
4812.35,4824.13,4836.22,4848.24,4864.32,4876.41 \\
5232.50,5237.34,5274.99^{b}, 5280.08,5308.44,5310.70,5313.59,5334.88,5337.79^{b} \\
5502.05^{b, c}, 5508.60 \\
6053.48,6226.66\end{array}$ \\
\hline Fe II & $\begin{array}{c}34 \\
35 \\
36 \\
40 \\
41 \\
42 \\
46 \\
48 \\
49 \\
55 \\
57 \\
72 \\
73 \\
74 \\
163 \\
182 \\
199 \\
200 \\
\text { uncl. }\end{array}$ & $\begin{array}{l}6217.95,6219.54,6229.34,6239.36 \\
5100.55,5120.34 \\
4993.36,5036.92 \\
6369.45^{b}, 6432.65^{a}, 6516.05^{a} \\
5256.89,5284.09 \\
4923.92,5018.43^{a}, 5169.03 \\
5991.38^{a}, 6044.53,6084.11^{a}, 6113.32,6116.04 \\
5264.80,5337.71^{b}, 5362.86,5414.09 \\
5197.57^{a}, 5234.62,5254.92,5275.99^{b}, 5316.61^{a}, 5325.55,5346.56,5425.27,5477.67 \\
5432.98,5534.86^{a}, 5591.38 \\
5627.49,5657.92,5725.95 \\
7289.05^{b}, 7533.42^{a} \\
7222.39^{a}, 7307.97^{a}, 7320.70^{a}, 7462.38^{a}, 7515.88^{a}, 7711.71^{a} \\
6147.73^{b}, 6149.24^{b}, 6238.38^{a}, 6247.56^{a}, 6407.30^{a}, 6416.90^{a}, 6456.38^{a} \\
6179.38 \\
5952.49 \\
6331.97,6446.43 \\
6103.54,6175.16,6305.32 \\
5387.14,5427.83,5466.02,5503.40,5510.78,5588.15,5645.40,5962.40,6157.12 \\
6317.99,6383.75,6385.47,6396.31,6442.95,6491.28,6623.07,6644.25,6729.86 \\
8489.69,8722.14\end{array}$ \\
\hline $\mathrm{H}$ & $\begin{array}{c}9 \\
10\end{array}$ & $\begin{array}{l}8598.39(\mathrm{~Pa} 14), 8665.02(\mathrm{~Pa} 13), 8750.47(\mathrm{~Pa} 12), 8862.79^{a}(\mathrm{~Pa} 11), 9014.91^{a}(\mathrm{~Pa} 10) \\
8413.32(\mathrm{~Pa} 19), 8437.96(\mathrm{~Pa} 18), 8467.25^{b}(\mathrm{~Pa} 17), 8502.49(\mathrm{~Pa} 16), 8545.38(\mathrm{~Pa} 15)\end{array}$ \\
\hline Mg I & 2 & $5167.32,5172.68,5183.60$ \\
\hline Mg II & $\begin{array}{c}8 \\
23\end{array}$ & $\begin{array}{l}7877.13,7896.37^{f} \\
6545.97^{c}\end{array}$ \\
\hline $\mathrm{N} \mathrm{I}$ & $\begin{array}{l}1 \\
2 \\
3 \\
8 \\
21 \\
30 \\
31 \\
40\end{array}$ & $\begin{array}{l}8680.24^{b, e}, 8683.38^{b}, 8686.13^{b}, 8703.24^{e}, 8711.69^{e}, 8718.82^{e}, 8728.88^{e}, 8747.35 \\
8184.80,8187.95,8200.31,8210.63,8216.28,8223.07^{b}, 8242.34 \\
7423.63,7442.28,7468.29 \\
8567.74,8594.01^{b}, 8629.24,8655.88^{b} \\
6481.71^{b}, 6482.70^{b}, 6483.75^{b}, 6484.80^{b}, 6506.45 \\
6758.60 \\
6706.20,6723.12,6733.48 \\
6752.40\end{array}$ \\
\hline OI & $\begin{array}{l}1 \\
4 \\
\end{array}$ & $\begin{array}{l}7771.96^{b}, 7774.18^{b}, 7775.40^{b} \\
8446.35^{a}\end{array}$ \\
\hline S II & $\begin{array}{c}6 \\
11\end{array}$ & $\begin{array}{l}5643.76^{c} \\
5578.85,5616.63\end{array}$ \\
\hline Sc II & $\begin{array}{l}21 \\
28 \\
29 \\
\end{array}$ & $\begin{array}{l}5854.31^{c} \\
6245.63 \\
5640.97,5669.03,5684.19 \\
\end{array}$ \\
\hline Si II & $\begin{array}{c}2 \\
4 \\
5 \\
\text { uncl. }\end{array}$ & $\begin{array}{l}6347.09^{e}, 6371.36^{b} \\
5957.61,5978.97 \\
5041.06,5056.02 \\
6660.52,6665.00,6671.88,6699.38\end{array}$ \\
\hline Ti II & $\begin{array}{c}69 \\
70 \\
86 \\
92 \\
103 \\
109 \\
112 \\
114 \\
298 \\
\end{array}$ & $\begin{array}{l}5336.81,5381.02,5418.80 \\
5188.70,5226.53,5262.10 \\
5129.14,5185.90 \\
4805.11 \\
5268.62 \\
5473.52 \\
6717.91,6680.26 \\
4874.02,4911.21 \\
5259.98\end{array}$ \\
\hline V II & $\begin{array}{c}55 \\
97 \\
238 \\
239\end{array}$ & $\begin{array}{l}5240.97,5271.26,5280.62 \\
6028.26^{d} \\
5642.01^{c} \\
5341.22^{d}\end{array}$ \\
\hline
\end{tabular}

${ }^{\dagger}$ Multiplet from Moore (1945). Lines that are unclassified are marked as “uncl.”; ${ }^{a}$ inverse P Cygni profile in 21/09/2003 spectrum; ${ }^{b}$ blended with nearby line; ${ }^{c}$ uncertain; ${ }^{d}$ highly uncertain, very weak; ${ }^{e}$ excess absorption apparant on blue wing; ${ }^{f}$ possible P Cygni profile. 


\section{References}

Andrillat, Y., Jaschek, M., \& Jaschek, C. 1996, A\&AS, 118, 495 Bonanos, A. Z. 2007, AJ, 133, 2696

Borgman, J., Koornneef, J., \& Slingerland, J. 1970, A\&A, 4, 248 Bowen, I. S. 1947, PASP, 59, 196

Bray, I., Burgess, A., Fursa, D. V., \& Tully, J. A. 2000, A\&AS, 146, 481

Chentsov, E. L., Ermakov, S. V., Klochkova, V. G., et al. 2003, A\&A, 397, 1035

Clark, J. S., \& Negueruela, I. 2004, A\&A, 413, L15

Clark, J. S., Negueruela, I., Crowther, P. A., \& Goodwin, S. 2005, A\&A, 434, 949

Clark, J. S., Muno, M. P., Negueruela, I., et al. 2008, A\&A, 477, 147

Clark, J. S., Ritchie, B. W., \& Negueruela, I. 2009, A\&A, submitted

Crowther, P. A., Hadfield, L. J., Clark, J. S., Negueruela, I., \& Vacca, W. D. 2006, MNRAS, 372, 1407

Damineli, A. 2001 in Eta Carinae and Other Mysterious Stars: the Hidden Opportunites of Emission Spectroscopy, ed. T. R. Gull, S. Johansson, \& K. Davidson (San Fransisco: ASP), ASP Conf. Proc., 242, 203

Davies, B., Oudmaijer, R. D., \& Vink, J. S. 2005, A\&A, 439, 1107

Davies, B., Oudmaijer, R. D., \& Sahu, K. C. 2007, ApJ, 671, 2059 de Jager, C. 1998, AARv, 8, 145

de Jager, C., \& Nieuwenhuijzen, H. 1997, MNRAS, 290, L50

Dekker, H., Delabre, B., \& D'Odorico, S. 1986, SPIE, 627, 39

Dekker, H., D’Odorico, S., Kaufer, A., Delabre, B., \& Kotzlowski, H. 2000, SPIE, 4008, 534

Dougherty, S. M., Clark, J. S., Negueruela, I., Johnson, T., \& Chapman, J. M. 2009, A\&A, submitted

Eikenberry, S. S., Matthews, K., LaVine, J. L., et al. 2004, ApJ, 616, 506

Figer, D. F., Najarro, F., Morris, M., et al. 1998, ApJ, 506, 384

Gal-Yam, A., \& Leonard, D. C. 2009, Nature, 458, 865

Grandi, S. A. 1980, ApJ, 238, 10

Groh, J. H., Damineli, A., Teodoro, M., \& Barbosa, C. L. 2006, A\&A, 457, 591

Groh, J. H., Damineli, A., \& Jablonski, F. 2007, A\&A, 465, 993

Groh, J. H., Hillier, D. J., Damineli, A., et al. 2009, ApJ, 698, 1698

Hamann, F., \& Simon, M. 1988, ApJ, 327, 867

Hillier, D. J., \& Miller, D. L. 1998, ApJ, 496, 407

Hillier, D. J., \& Miller, D. L. 1999, ApJ, 519, 354

Hillier, D. J., Crowther, P. A., Najarro, F., \& Fullerton, A. W. 1998, A\&A, 340, 483

Howarth, I. D., Murray, J., Mills, D., \& Berry, D. S. 2003, in Starlink User Note 50.24, Rutherford Appleton Laboratory

Humphreys, R. M., \& Davidson, K. 1994, PASP, 106, 1025

Humphreys, R. M., Davidson, K., \& Smith, N. 2002, ApJ, 124, 1026

Jenniskens, P., \& Desert, F.-X. 1994, A\&AS, 106, 39

Jones, T. J., Humphreys, R. M., Gehrz, R. D., et al. 1993, ApJ, 411, 323

Johansson, S. 1984, Phys. Scripta, T8, 63

Koorneef, J. 1977, A\&A, 55, 469

Kwan, J. 1984, ApJ, 283, 70

Lamers, H. J. G. L. M., Zickgraf, F.-J., de Winter, D., Houziaux, L., \& Zorec, J. 1988, A\&A, 340, 117
Lobel, A., Israelian, G., de Jager, C., et al. 1998, A\&A, 330, 659

Lobel, A., Dupree, A. K., Stefanik, R. P., et al. 2003, ApJ, 583, 923

Lockwood, G. W. 1974, ApJ, 193, 103

Machado, M. A. D., de Araújo, F. X., Pereira, C. B., \& Fernandes, M. B. 2002, A\&A, 387, 151

Moore, C. E. 1945, A multiplet table of astrophysical interest, Contribution from the Princeton University Observatory, 20

Munari, U., \& Tomasella, L. 1999, A\&AS, 137, 521

Munari, U., Siviero, A., Bienaymé, O., et al. 2009, A\&A, 503, 511

Muno, M. P., Bower, G. C., Burgasser, A. J., et al. 2006, ApJ, 638, 183

Najarro, F., Figer, D. F., Hillier, D. J., Geballe, T. R., \& Kudritzki, R. P. 2009, ApJ, 691, 1816

Nielsen, K. E., Corcoran, M. F., Gull, T. R., et al. 2007, ApJ, 660, 669

Nota, A., Smith, L., Pasquali, A., Clampin, M., \& Stroud, M. 1997, ApJ, 486, 338

Osterbrock, D. E., \& Ferland, G. J. 2006, in Astrophysics of Gaseous Nebulae and Active Galactic Nuclei, second edition (Sausalito, California: Univ. Science Books)

Oudmaijer, R. D. 1998, A\&AS, 129, 541

Oudmaijer, R. D., Geballe, T. R., Waters, L. B. F. M., \& Sahu, K. C. 1994, A\&A, 281, L33

Oudmaijer, R. D., Davies, B., de Wit, W.-J., \& Patel, M. 2009, in Biggest, Baddest, Coolest Stars, ed. D. G. Luttermoser, B. J. Smith \& R. E. Stencel, ASP Conf. Ser., 412, 17

Pasquani, L., Avila, G., Blecha, A., et al. 2002, The Messenger, 110, 1

Prieto, J. L., Kistler, M. D., Thompson, T. A., et al. 2008, ApJ, 681, L9

Ritchie, B. W., Clark, J. S., Negueruela, I., \& Crowther, P. A. 2009, A\&A, 507, 1585

Schaller, G., Schaerer, D., Meynet, G., \& Maeder, A. 1992, A\&AS, 96, 269

Shields, J. C., Pogge, R. W., \& De Robertis, M. M. 1999, in Structure and Kinematics of Quasar Broad Line Regions, ASP Conf. Ser., 175, 353

Shortridge, K., Meyerdicks, H., Currie, M., et al. 1997, in Starlink User Note 86.15, Rutherford Appleton Laboratory

Sigut, T. A. A., \& Pradhan, A. K. 1998, ApJ, 499, L139

Smith, N. 2007, AJ, 133, 1034

Smith, N., Vink, J. S., \& de Koter, A. 2004, ApJ, 615, 475

Smith, N., Li, W., Foley, R. J., et al. 2007, ApJ, 666, 1116

Stahl, O., Gäng, T., Sterken, C., et al. 2003, A\&A, 400, 279

Stickland, D. J., \& Harmer, D. L. 1978, A\&A, 70, L53

van Helden, R. 1972, A\&A, 19, 388

Verner, E. M., Gull, T. R., Bruhweiler, F., et al. 2002, ApJ, 581, 1154

Walborn, N. R., Stahl, O., Gamen, R. C., et al. 2008, ApJ, 683, 33

Weis, K. 2003, A\&A, 408, 205

Westerlund, B. E. 1961, PASP, 73, 51

Westerlund, B. E. 1987, A\&AS, 70, 311

Wolf, B. 1992, RvMA, 5, 1

Wolf, B., \& Stahl, O. 1990, A\&A, 235, 340 